\title{
VERY SINGULAR SIMILARITY SOLUTIONS AND HERMITIAN SPECTRAL THEORY FOR SEMILINEAR ODD-ORDER PDES
}

\author{
R.S. FERNANDES AND V.A. GALAKTIONOV
}

\begin{abstract}
Asymptotic large- and short-time behaviour of solutions of the linear dispersion equation

$$
u_{t}=u_{x x x} \quad \text { in } \quad \mathbb{R} \times \mathbb{R}_{+},
$$
\end{abstract}

and its $(2 k+1)$ th-order extensions are studied. Such a refined scattering is based on a "Hermitian" spectral theory for a pair $\left\{\mathbf{B}, \mathbf{B}^{*}\right\}$ of non self-adjoint rescaled operators

$$
\mathbf{B}=D_{y}^{3}+\frac{1}{3} y D_{y}+\frac{1}{3} I, \text { and the adjoint one } \mathbf{B}^{*}=D_{y}^{3}-\frac{1}{3} y D_{y}
$$

with the discrete spectrum $\sigma(\mathbf{B})=\sigma\left(\mathbf{B}^{*}\right)=\left\{\lambda_{l}=-\frac{l}{3}, l=0,1,2, \ldots\right\}$ and eigenfunctions $\left\{\psi_{l}(y)=\frac{(-1)^{l}}{\sqrt{l !}} D_{y}^{l} \operatorname{Ai}(y), l \geq 0\right\}$, where $\operatorname{Ai}(y)$ is Airy's classic function. Applications to very singular similarity solutions (VSSs) of the semilinear dispersion equation with absorption,

$$
u_{\mathrm{S}}(x, t)=t^{-\frac{1}{p-1}} f\left(\frac{x}{t^{1 / 3}}\right): \quad u_{t}=u_{x x x}-|u|^{p-1} u \quad \text { in } \quad \mathbb{R} \times \mathbb{R}_{+}, \quad p>1,
$$

and to its higher-order counterparts are presented. The goal is, by using various techniques, to show that there exists a countable sequence of critical exponents $\left\{p_{l}=\right.$ $\left.1+\frac{3}{l+1}, l=0,1,2, \ldots\right\}$ such that, at each $p=p_{l}$, a $p$-branch of VSSs bifurcates from the corresponding eigenfunction $\psi_{l}$ of the linear operator $\mathbf{B}$ above.

\section{INTRODUCTION: SEMILINEAR ODD-ORDER MODELS, HISTORY, AND RESULTS}

1.1. Basic dispersion models and applications. As a first basic model, we will study higher odd-order partial differential equations (PDEs) of the form

$$
u_{t}=(-1)^{k+1} D_{x}^{2 k+1} u+\tilde{g}(u) \quad \text { in } \quad \mathbb{R} \times \mathbb{R}_{+}, \quad k=1,2, \ldots,
$$

with bounded integrable initial data $u(x, 0)=u_{0}(x)$ in $\mathbb{R}$. Here $D_{x}^{m}=(\partial / \partial x)^{m}$ denotes the $m^{\text {th }}$ partial derivative in $x$. The odd-order semilinear dispersion equation (1.1) can be considered as a counterpart of the better known semilinear higher-even-order parabolic PDE of reaction-diffusion type,

$$
u_{t}=(-1)^{k+1} D_{x}^{2 k} u+\tilde{g}(u) \quad \text { in } \quad \mathbb{R} \times \mathbb{R}_{+}, \quad k=1,2, \ldots .
$$

Date: November 5, 2018

1991 Mathematics Subject Classification. 35K55, 35K40.

Key words and phrases. Odd-order linear and semilinear PDEs, fundamental solution, Hermitian spectral theory, polynomial eigenfunctions, self-similarity, very singular solutions, bifurcations, branching. 
For $k=1$, (1.2) becomes a standard reaction-diffusion equation from combustion theory

$$
u_{t}=u_{x x}+\tilde{g}(u) \text { in } \mathbb{R} \times \mathbb{R}_{+},
$$

to which dozens of well-known monographs are devoted to. These parabolic equations indeed belongs to an entirely different type of PDEs and were much better studied in the twentieth century. However, the analogy between odd and even-order PDEs, such as (1.1) and (1.2), is rather fruitful and will be used later on.

The function (a nonlinear operator) $\tilde{g}(u)$ in (1.2) usually corresponds to some absorptionreaction type phenomena and sometimes is assumed to include differential terms, such as $D_{x}^{m} u$, with $m<2 k+1$ (although we do not consider such cases). It is worth mentioning again that, besides some special and completely integrable PDEs, general odd-order models such as (1.1) are less studied in the mathematical literature, than the parabolic even-order ones (1.2).

Indeed, the most classical example of such an odd-order equation is the $K d V$ equation:

$$
u_{t}=u_{x x x}+u u_{x} \quad\left(\tilde{g}(u):=u u_{x}\right),
$$

which was introduced by Boussinesq in 1872 together with its soliton solution [5]. The $\mathrm{KdV}$ equation models long waves in shallow water and generates a hierarchy of other more complicated PDEs with linear and nonlinear dispersion (dispersive) mechanisms. See further amazing historical aspects concerning (1.4) and related integrable PDEs in [25, pp. 226-229].

Concerning higher-order extensions, these naturally appear in classic theory of integrable PDEs from shallow water applications, including the fifth-order KdV equation,

$$
u_{t}+u_{x x x x x}+30 u^{2} u_{x}+20 u_{x} u_{x x}+10 u u_{x x x}=0 .
$$

Let us also mention such classic examples as Lax's seventh-order KdV equation

$$
u_{t}+\left[35 u^{4}+70\left(u^{2} u_{x x}+u\left(u_{x}\right)^{2}\right)+7\left(2 u u_{x x x x}+3\left(u_{x x}\right)^{2}+4 u_{x} u_{x x x}\right)+u_{x x x x x x}\right]_{x}=0,
$$

and the seventh-order Sawada-Kotara equation

$$
u_{t}+\left[63 u^{4}+63\left(2 u^{2} u_{x x}+u\left(u_{x}\right)^{2}\right)+21\left(u u_{x x x x}+\left(u_{x x}\right)^{2}+u_{x} u_{x x x}\right)+u_{x x x x x x}\right]_{x}=0 ;
$$

see [25, p. 234] for references and other odd-order models from compacton theory.

Of course, PDEs such as (1.4) and (1.1) were popular in mathematical physics last fifty years at least (see references below), are have been well-known in mathematical theory. We refer to a number of papers on local and global existence, uniqueness, smoothing, and various asymptotic (scattering-like) properties; see as a guide [7, 10, 11, 12, 28, 30, 31, 40, 42, 45, 53, 54, where some papers on linear Schrödinger operators that exhibit related aspects of smoothing and evolution are also included. However, for our purposes of studying refined asymptotic properties of (1.1) (a refined scattering theory), these strong results turn out to be not quite enough. Therefore, first of all, we will need to develop new Hermitian spectral theory of linear rescaled odd-order operators, which will refine some necessary delicate asymptotic properties of PDEs under consideration. 
In particular, as a first unavoidable step, we will need to study in detail the longand short-time (on blow-up micro-scales) behaviour of solutions of the simplest linear dispersion equation for $k=1$ (the LDE-3)

$$
\text { Basic Model I : } u_{t}=u_{x x x} \text { in } \mathbb{R} \times \mathbb{R}_{+},
$$

which, even in this simple standard form, will create a number of surprises that require difficult unusual "spectral" techniques. As a result, we then will be able to study similarity solutions ("nonlinear eigenfunctions") of the semilinear dispersion equation

$$
\text { Basic Model II : } u_{t}=u_{x x x}-|u|^{p-1} u \quad \text { in } \quad \mathbb{R} \times \mathbb{R}_{+}, \quad \text { where } \quad p>1 .
$$

These two PDEs (1.5) and (1.6) are our main simplest canonical models to study.

1.2. First similarity solutions: earlier history and Prandtl-Blasius results for odd-order boundary layer problem. For both semilinear (1.2) and linear (1.5) oddorder equations, we will apply the idea of self-similar solutions. In general, there are several techniques of solving PDEs, including using travelling wave and other groupinvariant solutions. As customary, similarity solutions are a common way of attempting to understand and sometimes solve some problems. Similarity solutions are widely used in PDE theory, since they simplify the problem by transforming PDEs into much simpler ODEs, and hence this reduces the number of independent variables. Rescaled variables can be used in studying PDEs, since the coordinate system in which the problem is posed does not affect the formulation of any fundamental physical laws involved.

The first general type of explicit solutions was traveling waves in eighteen's century d'Alembert's formula for the linear wave equation. The method of separation of variables was developed by Fourier in the study of heat conduction problems, and was later generalized and extended by Sturm and Liouville in the 1830s. Many famous mathematicians, such as Euler, Poisson, Lagrange, Liouville, Sturm, Laplace, Darboux, Bäcklund, Lie, Jacobi, Boussinesq, Goursat, and others developed various techniques for obtaining explicit solutions of a variety of linear and nonlinear models from physics and mechanics. Their methods included a number of particular transformations, symmetries, expansions, separation of variables, etc. Similarity solutions appeared in the works by Weierstrass around 1870, and by Bolzman around 1890. General principles for finding solutions of systems of ODEs and PDEs by symmetry reductions date back to the famous Lie papers published in the 1880s and 1890s.

More complicated and of key importance similarity solutions next appeared in Prandtl's equation, occurring in Prandtl's boundary layer theory, which was proposed in 1904, [48. After that, similarity solutions of linear and nonlinear boundary-value problems became more common in the literature. Namely, this similarity solution in $\mathbb{R}^{2}$ was due to Blasius (1908) [4] for the equation of incompressible fluids,

$$
\psi_{y y y}+\psi_{x} \psi_{y y}-\psi_{y} \psi_{x y}-\psi_{y t}+u u_{x}+U_{t}=0
$$

where $\psi=\psi(x, y, t)$ is the stream function and $U(x, t)$ is the given external far-field (at $y=\infty)$ velocity distribution. Remarkably, this is an odd-order PDE. 
1.3. On even-order models: the canonical heat equation. For a long period, until 1980s, whilst many asymptotic and singularity aspects of higher odd-order models have not been studied in great detail, for a number of years, various even-order, both lower and higher order, models have been reasonably well understood. The basic linear and most classical case of such a PDE is the heat equation. It is one of the most important linear partial differential equations. A natural logic of the first part of our research of the LDE-3 (1.5) can be conveniently connected with this classic area.

Thus, the one-dimensional heat equation is the canonical PDE

$$
u_{t}=u_{x x} \quad \text { in } \quad \mathbb{R} \times \mathbb{R}_{+} .
$$

It has the classic fundamental solution

$$
b(x, t)=\frac{1}{\sqrt{t}} F(y), \quad \text { with the similarity variable } y=\frac{x}{\sqrt{t}},
$$

which takes Dirac's delta as an initial function, i.e., in the sense of distributions, $b(x, 0)=$ $\delta(x)$. Substituting (1.9) into the heat equation (1.8) yields a simple ODE for the rescaled kernel $F$ :

$$
\mathbf{B} F \equiv F^{\prime \prime}+\frac{1}{2}(F y)^{\prime}=0 \text { in } \mathbb{R}, \quad \int F=1 .
$$

This is solved explicitly to show that the solution, $F$, is the Gaussian:

$$
F(y)=\frac{1}{2 \sqrt{\pi}} \mathrm{e}^{-\frac{y^{2}}{4}}
$$

which is strictly positive and does not have oscillatory components to be traced out for linear dispersion equations. Full spectral theory has been developed, with eigenvalues of the linear second-order operator $\mathbf{B}$ and eigenfunctions given by (see Birman-Solomjak [3, p. 48] for full details and spectral theory in $\mathbb{R}^{N}$ )

$$
\sigma(\mathbf{B})=\left\{\lambda_{l}=-\frac{l}{2}, l=0,1,2, \ldots\right\} \quad \text { and } \quad \psi_{l}(y)=\frac{(-1)^{l}}{\sqrt{l !}} D_{y}^{l} F(y) \equiv H_{l}(y) F(y) .
$$

Here $H_{l}(y)$ denote the standard orthonormal Hermite polynomials (introduced in full generality by Hermite in the 1870s), which are induced by the Gaussian (1.11). These Hermite polynomials in 1D were earlier derived by C. Sturm in 1836 [52, where these were used for a classification of all types of multiple spatial zeros for solutions $u(x, t)$ of the 1D heat equation. This led him to the now famous Sturm Theorems on zero set; see a full history in [17, Ch. 1]. The Sturmian results found for the linear heat equation, have been the basis for other higher and nonlinear even-order and other related models, where the zero set analysis of solutions become indeed more involved; see [18] and references therein. Whilst solutions to these models retain the basic symmetric Gaussian structure, they have some oscillatory tails instead of a pure simple exponential decay, which essentially change the meaning of Sturmian theorems.

Thus, the discrete spectrum and Hermite polynomials in (1.12) are the cornerstone of the Hermitian spectral theory of the rescaled operator $\mathbf{B}$ in (1.10). Since $\mathbf{B}$ admits a symmetric representation:

$$
\mathbf{B} \equiv \frac{1}{\rho} \frac{\mathrm{d}}{\mathrm{d} y}\left(\rho \frac{\mathrm{d}}{\mathrm{d} y}\right), \quad \underset{4}{\text { where }} \quad \rho(y)=\mathrm{e}^{\frac{y^{2}}{4}}
$$


this theory is essentially self-adjoint in the weighted space $L_{\rho}^{2}(\mathbb{R})$ including the $N$-dimensional case [3]. As an unavoidable step, we will need to develop a similar theory for the LDE-3 (1.5), which is not self-adjoint and will go in different lines than the classic one.

1.4. Very singular similarity solutions. Around the beginning of the 1980 s, study of asymptotics of the semilinear heat equation with absorption, given by

$$
u_{t}=\Delta u-u^{p} \quad \text { in } \quad \mathbb{R}^{N} \times \mathbb{R}, \quad p>1 \quad(u \geq 0),
$$

led to a new class of similarity solutions called very singular solutions (VSSs). These are self-similar solutions, which are structurally stable in the evolution and hence, as $t \rightarrow+\infty$, attract wide classes of other more general solutions. In addition, as $t \rightarrow 0$, such a VSS concentrates at $x=0$ with infinite initial mass and this justifies the term "very singular". We refer to the books [26, 49] for extra details and history concerning VSSs. Whilst VSSs were being studied early on, it wasn't until about 1985 in papers by Kamin and Peletier [6, 32] in which the term VSS was actually used.

The VSSs for the semilinear heat equation (1.13) are given by

$$
\begin{gathered}
u_{*}(x, t)=t^{-\frac{1}{p-1}} f(y), \quad y=\frac{x}{\sqrt{t}}, \quad \text { where } f>0 \text { solves the elliptic equation } \\
\left\{\begin{array}{l}
\Delta f+\frac{1}{2} y \cdot \nabla f+\frac{1}{p-1} f-f^{p}=0 \text { in } \mathbb{R}^{N}, \\
f(y) \text { has exponential decay as } y \rightarrow \infty .
\end{array}\right.
\end{gathered}
$$

Existence of the VSS was established in [22] using a PDE approach, and almost simultaneously, via an ODE approach, in [6]. Uniqueness of VSS was first proved later in [33], by using a PDE comparison method. We refer to [19, 27] for more references and history of VSSs for semilinear parabolic equation.

1.5. Back again to the $\mathbf{K d V}$-type equations. It is curious that, formally, VSSs can be prescribed for the classic KdV equation (1.4). These have a standard self-similar form

$$
u_{*}(x, t)=t^{-\frac{2}{3}} f(y), \quad y=x / t^{\frac{1}{3}} .
$$

Hence (1.4) reduces to the following ODE:

$$
f^{\prime \prime \prime}+\frac{1}{3} y f^{\prime}+\frac{2}{3} f+f f^{\prime}=0 \quad \text { in } \quad \mathbb{R} .
$$

However, whilst we can seemingly construct a VSS for the KdV equation, we know that solutions of this type do not exist. The only natural solution we arrive at is the trivial one, $f \equiv 0$. This nonexistence conclusion directly follows from the global existence results by Kato [35, 34] and Strauss [51]. Indeed, (1.4) is invariant under reflection with

$$
t \mapsto T-t \quad \text { and } \quad x \mapsto-x,
$$

where very singular solutions (1.14) are then given by

$$
u_{*}(x, t)=(T-t)^{-\frac{2}{3}} f(y), \quad y=x /(T-t)^{\frac{1}{3}} .
$$

Therefore existence of a nontrivial VSS would mean finite time blow-up of solutions, since

$$
\sup _{x}|u(x, t)| \sim(T-t)^{-\frac{2}{3}} \rightarrow+\infty \quad \text { as } \quad t \rightarrow T^{-} .
$$


In view of well-known global existence results for the KdV equation (see references above), this implies the non-existence of very singular solutions for (1.4).

For the modified KdV equation

$$
u_{t}=u_{x x x}+u^{p} u_{x}
$$

we can find VSS for $p \geq 4$, but not for $p<4$. In the case $p \geq 4$, (1.17) has self-similar solutions of the standard form

$$
u_{*}(x, t)=t^{-\frac{2}{3 p}} f(y), \quad y=x / t^{\frac{1}{3}},
$$

where the rescaled kernel satisfies

$$
f^{\prime \prime \prime}+\frac{1}{3} y f^{\prime}+\frac{2}{3 p} f+f^{p} f^{\prime}=0 .
$$

The case $p=4$ has been studied more extensively; see papers [36, 9] as just two examples. We thus need to study such similarity solutions for our second semilinear model (1.6).

1.6. Four main linear and nonlinear odd-order models to study. We will treat, in particular, three generalized odd-order models, which, from our "spectral-like" and refined asymptotic points of view, essentially have never been looked at before. In particular, we look for similarity solutions and using asymptotic, analytic, and some numerical methods, we will attempt to find and justify some local and global properties of the rescaled solutions. We use a number of techniques, applied previously for parabolic even-order problems, for the odd-order ones. However, there are difficulties that arise, in particular due to the highly oscillatory nature of fundamental solutions and hence related linear and nonlinear eigenfunctions.

Our FIRST AIM is to develop a Hermitian spectral theory of the associated rescaled operators, for linear dispersion odd-order equations such as (cf. (1.5) for $k=1$ )

$$
\text { (I) : } \quad u_{t}=(-1)^{k+1} D_{x}^{2 k+1} u \quad \text { in } \quad \mathbb{R} \times \mathbb{R}_{+} \text {for any } k \geq 1 .
$$

In doing this, we gain the SECOND GOAL, an understanding of its behaviour, which we can then use in the corresponding semilinear dispersion equation and its VSSs

$$
\text { (II) : } \quad u_{t}=(-1)^{k+1} D_{x}^{2 k+1} u-|u|^{p-1} u \quad \text { in } \quad \mathbb{R} \times \mathbb{R}_{+}, \quad \text { where } \quad p>1 .
$$

In a forthcoming paper [15], these concepts and ideas will be applied and extended to PDEs with nonlinear dispersion (the NDEs) of the form

$$
(\text { III }): \quad u_{t}=(-1)^{k+1} D_{x}^{2 k+1}\left(|u|^{n} u\right) \quad \text { in } \quad \mathbb{R} \times \mathbb{R}_{+}, \quad \text { where } n>0 .
$$

Concerning self-similar solutions of (1.21), called "nonlinear eigenfunctions", asymptotic behaviour and general properties of solutions such as existence, uniqueness, shock waves, entropy approaches, etc., turned out to create a number of very difficult questions. First steps of the study of shock and rarefaction waves for NDEs including the NDE-3 such as

$$
u_{t}=\left(u u_{x}\right)_{x x} \text { in } \mathbb{R} \times \mathbb{R}_{+},
$$

are performed in [20, 24]. As a natural extension of the NDE (1.21), we also discuss the VSS for the full NDE:

$$
(\mathbf{I V}): \quad u_{t}=(-1)^{k+1} D_{x}^{2 k+1}\left(|u|^{n} u\right)-|u|^{p-1} u \quad \text { in } \quad \mathbb{R} \times \mathbb{R}_{+}, \quad \text { where } \quad p>n+1
$$


In both cases of NDEs (1.21) and (1.22), the key idea is to perform a "homotopy" limit $n \rightarrow 0^{+}$to arrive at the rescaled linear operators and next to use Hermitian spectral theory for bifurcation-branching analysis of nonlinear eigenfunctions and VSSs.

\section{BASIC LINEAR DISPERSION MODELS: LDES AND THEIR FUNDAMENTAL SOLUTIONS}

2.1. Basic LDE: fundamental solutions and rescaled kernels. Before looking at some more complicated nonlinear PDEs, it is important to understand in greater details how the solutions of linear PDEs behave for large and small times. This spectral theory of higher-odd-order linear rescaled operators will be crucial in the understanding of related nonlinear ones. In particular, as customary, spectral theory formed in the linear case will play a large role and will be used in developing understanding of bifurcations, branching, and asymptotic behaviour for nonlinear equations.

Thus, we consider the corresponding linear dispersion equation (1.19), which we al so call the LDE- $(2 k+1)$. Whilst some lower-order cases for the odd-order linear PDE (1.19) such as the LDE-3 (1.5) are generally better understood, the higher-order cases for $k \gg 1$ are not. Indeed, it is well-known that the fundamental solution for the lowest order case $k=1$, i.e., for (1.5), will lead to the classic Airy function. However, we will need a more general calculus that is applied for arbitrary $k \geq 1$.

Consider the self-similar fundamental solution of (1.19) of the standard similarity form

$$
b(x, t)=t^{-\frac{1}{2 k+1}} F(y), \quad y=x / t^{\frac{1}{2 k+1}}, \quad \text { so that } \quad b(x, 0)=\delta(x),
$$

in the sense of distributions. Substituting $b(x, t)$ into the PDE (1.19), we obtain the ODE for the rescaled kernel $F$,

$$
\mathbf{B} F \equiv(-1)^{k+1} D_{y}^{2 k+1} F+\frac{1}{2 k+1} y D_{y} F+\frac{1}{2 k+1} F=0 \quad \text { in } \quad \mathbb{R}, \quad \int F=1,
$$

where $\mathbf{B}$ denotes the first key linear rescaled operator for the LDE. Note that it is possible to integrate the ODE (2.2) once, to find that $F(y)$ solves

$$
(-1)^{k+1} F^{(2 k)}+\frac{1}{2 k+1} y F=0 \text { for } y \in \mathbb{R},
$$

which is now a linear ODE of order $2 k$. Further reductions of this ODE are not possible.

For $k=1$, the solution of (2.3) is the classic Airy function:

$$
F^{\prime \prime}+\frac{1}{3} y F=0 \quad \Longrightarrow \quad F(y)=\operatorname{Ai}(y) .
$$

Note that in our case, due to a difference in sign in the ODE, we actually have $\operatorname{Ai}(-y)$, but we shall refer to it as the Airy one.

2.2. Asymptotic expansions of the rescaled fundamental kernel. An important and standard technique in trying to find the behaviour of solutions of ODEs is to use asymptotic analysis. This gives the limiting behaviour of solutions, in particular as $y \rightarrow$ $\pm \infty$. We refer to the book by Bender and Orszag [2] for various asymptotic techniques. We use here a method of determining the asymptotic behaviour of the linear ODE, which corresponds to the classic WKBJ multi-scale analysis of ODEs, whose basic ideas go back to the 1920s and were reflected in a number of well-known monographs. Actually, in 
ODE theory, asymptotics for ODEs such as (2.3) are well-known and have been classified. However, we will need some more refined formulae for further applications. Some of them for arbitrary $k \geq 1$ are not available in standard literature. Looking at the asymptotic behaviour of the solution of the integrated ODE (2.3), we now write it as

$$
(-1)^{k+1} F^{(2 k)}=-\frac{1}{2 k+1} F y .
$$

Algebraic decay as $y \rightarrow+\infty$. Let us look first at the rescaled solution $F(y)$ with oscilla-

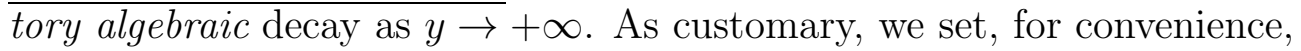

$$
F(y)=\mathrm{e}^{s(y)} \quad \text { as } \quad y \rightarrow \infty .
$$

Now assume, as a first approximation, that $s(y)$ is some polynomial, such that $s(y) \sim a y^{b}$. Then the kernel $F(y)$ and its derivatives may be given by

$$
\begin{gathered}
F^{\prime}=s^{\prime} \mathrm{e}^{s}, \quad F^{\prime \prime}=\left[s^{\prime \prime}+\left(s^{\prime}\right)^{2}\right] \mathrm{e}^{s}, \quad F^{\prime \prime \prime}=\left[s^{\prime \prime \prime}+3 s^{\prime} s^{\prime \prime}+\left(s^{\prime}\right)^{3}\right] \mathrm{e}^{s}, \ldots, \\
F^{(2 k)}=\left[s^{(2 k)}+\ldots+k(2 k-1)\left(s^{\prime}\right)^{2 k-2} s^{\prime \prime}+\left(s^{\prime}\right)^{2 k}\right] \mathrm{e}^{s},
\end{gathered}
$$

where $s^{\prime} \sim a b y^{b-1}, s^{\prime \prime} \sim a b(b-1) y^{b-2}, \ldots, s^{(2 k)} \sim \frac{a b !}{(b-2 k) !} y^{b-2 k}$. Substituting this into the ODE (2.5), it can easily be seen that all the $\mathrm{e}^{s(y)}$ terms cancel. From the resulting equation, we look to do a dominant balance analysis, in order to determine the leading order behaviour of $F(y)$. In doing so, we find a first approximation for the function $s(y)$. The balance of the equation depends on the value of the parameter $b$ and we obtain two different cases.

If $b \leq 0$, then as $y \rightarrow+\infty$, every term on the left-hand side of the ODE is $o(y)$. Therefore there is no balance in this case. If $b>0$, then $y^{b-\tilde{n}}=o\left(y^{2 k(b-1)}\right)$, for any $\tilde{n}>1$. Therefore balancing leading terms, we have that

$$
(-1)^{k+1} a^{2 k} b^{2 k} y^{2 k(b-1)} \sim-\frac{1}{2 k+1} y,
$$

for all $k \in \mathbb{Z}_{+}$. By first equating powers of $y$ and then coefficients, we find our parameters

$$
b=\frac{2 k+1}{2 k} \quad \text { and } \quad a=-\mathrm{i}(2 k+1)^{-\frac{1}{2 k}}\left(\frac{2 k}{2 k+1}\right) .
$$

Hence, we now have the first approximation to $s(y)$, with

$$
\begin{gathered}
s(y)=-2 k \mathrm{i}\left(\frac{y}{2 k+1}\right)^{\frac{2 k+1}{2 k}}+c(y), s^{\prime}(y)=-\mathrm{i}\left(\frac{y}{2 k+1}\right)^{\frac{1}{2 k}}+c^{\prime}(y), \\
s^{\prime \prime}(y)=-\frac{\mathrm{i}}{2 k(2 k+1)^{\frac{1}{2 k}}} y^{-\frac{2 k-1}{2 k}}+c^{\prime \prime}(y), \ldots .
\end{gathered}
$$

Here $c(y) \sim o\left(y^{\frac{2 k+1}{2 k}}\right)$ is some function of $y$ and the next term in the approximation of $s(y)$. Since $k \geq 1$, we must have that $c^{(m+1)}(y) \sim o\left(c^{(m)}(y)\right)$ for any $m>1$, which will be used in determining leading order terms.

We attempt to find this function $c(y)$, in order to improve the approximation of $s(y)$. We let $g(y)=-2 k \mathrm{i}\left(\frac{y}{2 k+1}\right)^{\frac{2 k+1}{2 k}}$ for convenience, to see how the terms are balanced. Hence balancing the leading order terms yields $c^{\prime}(y) \sim-\frac{2 k-1}{2} \frac{g^{\prime \prime}(y)}{g^{\prime}(y)}=-\frac{2 k-1}{4 k y}$. Integrating this, the next term in the expansion can be found to be $c(y) \sim-\frac{2 k-1}{4 k} \ln y$. The second term in 
this expansion, $c(y)$, is called the controlling factor. It is an important term, as we will see that it governs the decay (or any possible growth) of solutions as $y \rightarrow+\infty$.

Whilst with the first two terms, we can have a clear idea of the leading order behaviour, we look to find a better approximation and now expand once again with

$$
s(y)=-2 k \mathrm{i}\left(\frac{y}{2 k+1}\right)^{\frac{2 k+1}{2 k}}-\frac{2 k-1}{4 k} \ln y+d(y),
$$

where $d(y)=o(c(y))$. Balancing leading order terms once again, we find that $d^{\prime}(y) \sim$ $-\frac{2 k-1}{4 k^{2} y^{2}}$. Then by integrating, we find that the third term in the expansion is given by $d(y) \sim \frac{2 k-1}{4 k^{2} y}$. Note that this is the third term in the expansion and hence all following lower-order terms are $o(1)$, and so, by (2.6), this does not affect the exponential.

From this, we find that the required asymptotic behaviour of $F(y)$ is given by

$$
F(y) \sim y^{-\frac{2 k-1}{4 k}} \exp \left\{-2 k \mathrm{i}\left(\frac{y}{2 k+1}\right)^{\frac{2 k+1}{2 k}}\right\} \quad \text { as } \quad y \rightarrow+\infty .
$$

Therefore, for real solutions, we have that, for some constant $\hat{c} \in \mathbb{R}$,

$$
F(y) \sim y^{-\frac{2 k-1}{4 k}} \cos \left(d_{k} y^{\frac{2 k+1}{2 k}}+\hat{c}\right) \quad \text { as } \quad y \rightarrow+\infty, \quad \text { where } \quad d_{k}=2 k\left(\frac{1}{2 k+1}\right)^{\frac{2 k+1}{2 k}} .
$$

One can see that (2.7) guarantees the convergence of the integral of $F$ in (2.2) (but not in the absolute sense), so that the fundamental solution $b(x, t)$ at $t=0$ takes Dirac's delta in the sense of bounded measures. Note that, a priori, this is not guaranteed in general, since delicate ODE asymptotics are necessary to charge such a property.

Exponential decay as $y \rightarrow-\infty$. The same analysis can be applied for $y \rightarrow-\infty$, by letting $\bar{y} \mapsto-y$ and performing the same calculations. Hence we find that the rescaled kernel decays exponentially fast in the opposite direction,

$$
F(y) \sim|y|^{-\frac{2 k-1}{4 k}} \cos \left(d_{k}|y|^{\frac{2 k+1}{2 k}} \sin b_{k}+\hat{c}\right) \exp \left\{-\hat{d}_{k}|y|^{\frac{2 k+1}{2 k}}\right\} \quad \text { as } \quad y \rightarrow-\infty,
$$

where $d_{k}$ is as in (2.7) and

$$
\hat{d}_{k}=d_{k}\left|\cos b_{k}\right|>0, \quad b_{k}=\left\{\begin{array}{l}
\frac{\pi}{k}\left\lfloor\frac{k+1}{2}\right\rfloor \quad \text { for even } k, \\
\frac{\pi}{k} \frac{k+1}{2}+\frac{\pi}{2 k} \quad \text { for odd } k .
\end{array}\right.
$$

We note that the coefficient $a$ has many roots, as follows from the algebraic equation:

$$
F(y) \sim \mathrm{e}^{a|y|^{(2 k+1) / 2 k}} \Longrightarrow a^{2 k}=\frac{(-1)^{k+1}(2 k)^{2 k}}{(2 k+1)^{2 k+1}},
$$

which represent different solutions of the ODE. However, we only want roots such that there is exponential decay, rather than growth. Therefore we exclude the roots where there is growth, which corresponds to $\operatorname{Re} a>0$. The asymptotics here show the behaviour of the first roots, such that Re $a \leq 0$. The method of finding these roots and all other roots may be seen in Appendix A, which also explains more carefully which roots we need to look at.

Thus, according to (2.7), as $y \rightarrow+\infty$, we have a purely imaginary root for all values of $k$ and this gives slow decaying oscillatory behaviour. The decay of the oscillations also increases for larger $k$, from $y^{-\frac{1}{4}}$ for $k=1$ towards $\sim y^{-\frac{1}{2}}$ as $k \rightarrow \infty$. On the contrary, as $y \rightarrow-\infty$, (2.8) shows exponential decay with oscillations for any $k>1$ and these 
oscillations are always exponentially fast. For the only case $k=1$, we have $b_{k}=\pi$, hence $\sin b_{k}=0$. This gives the pure exponential non-oscillatory behaviour of the Airy function:

$$
F(y) \sim|y|^{-\frac{1}{4}} \mathrm{e}^{-2 \cdot 3^{-\frac{3}{2}}|y|^{\frac{3}{2}}} \quad \text { as } \quad y \rightarrow-\infty .
$$

This is the reason why the Airy function is the only odd-order linear case where there is no exponential infinitely oscillatory behaviour as $y \rightarrow-\infty$. In a natural sense, this mimics the positive behaviour of the Gaussian (1.11), but only partially, in a one-sided limit $y \rightarrow-\infty$. Anyway, the exponential decay in (2.8) simplifies a part of spectral analysis of the operator $\mathbf{B}$, for which we will need to pay the main attention to the opposite limit $y \rightarrow+\infty$, where most of technical difficulties appeared from.

We now summarize the results of our asymptotic analysis to get a sharp uniform bound of the rescaled kernel $F$, which will eventually define the weighted functional space for $\mathbf{B}$ :

Proposition 2.1. The rescaled kernel $F(y)$ of the fundamental solution (2.1) of the LDE (1.19) satisfies

$$
|F(y)| \leq\left\{\begin{array}{l}
D_{0}\left(1+y^{2}\right)^{-\frac{2 k-1}{8 k}} \mathrm{e}^{-\hat{d}_{k}|y|^{\alpha}} \quad \text { for } y \leq 0 \\
D_{0}\left(1+y^{2}\right)^{-\frac{2 k-1}{8 k}} \quad \text { for } \quad y \geq 0,
\end{array}\right.
$$

where $\hat{d}_{k}$ is as in (2.9), $D_{0}$ is a positive constant dependent on $k$, and

$$
\alpha=\frac{2 k+1}{2 k} \in(1,2) \quad \text { for all } k \geq 1 \text {. }
$$

2.3. Numerical construction of fundamental kernels. The results here were obtained by using the MatLab bvp4c solver, to look at the singular solutions of the linear ODE (2.2).

Obviously, if $F$ is a solution of (2.3), then $c F$ is also a solution, for all $c \in \mathbb{R}$. Due to such "non-uniqueness" (and, to some extent, an "instability") of the solutions, the zero solution is likely to be found using numerical methods. Therefore in order to ensure that a non-zero solution is obtained, we set the (normalisation) constraint

$$
\max |F|=1 \text {, }
$$

which is attained at some point $y=\hat{a}$. We then solved the ODE for different right and left solutions at this maximum point $\hat{a}$, using the bvp4c solver. Since we have fixed $\hat{a}$ as a maximum, the first derivative is also zero at this point. The other boundary condition placed was to ensure that $F(y)=0$, at an end point that is sufficiently removed from $\hat{a}$. In order for a match of right and left solutions at the point $\hat{a}$, we needed to have the second derivative of the solution to be the same here, in order for $F(y)$ to be continuous at $y=\hat{a}$. So the value of $\hat{a}$ was moved in order to match the second derivative for the right and left solutions. See Figure 1, which was obtained by this shooting method.

Indeed, for $k=1$, as a simple alternative, one can use "shooting from the left" in the second-order ODE (2.4) by using the exponential decay asymptotics (2.10) for $y \ll-1$ to get a proper behaviour (2.7) for $y \gg 1$, since no other are available in the version (2.4). Such a simpler one-sided shooting is shown in Figure 2, which is rather similar to Figure 
(11) (b). What is most important is that the sizes of the tails in both Figures coincide. However, such an approach is hardly applied in higher-order cases $k \geq 2$, where extra solutions selecting procedures such as multi-dimensional shooting are unavoidable.

A similar method of two-sided shooting was applied to the fifth order $(k=2)$ equation, where values of the left-hand solution were used as boundary conditions for the right solution, and the correct value for $\hat{a}$ was found by matching the fourth derivative. See Figure 3. Note that, in view of (2.12), the plots do not show the fundamental solutions of the ODE, such that $\int F=1$, but rescaled profiles, for which the normalization (2.12) is satisfied.

As one can see from comparing Figures 1 and 3, the oscillations are smaller as $k$ increases. This follows from the asymptotic analysis done in Section 2.2. see (2.7). Decay of the algebraic envelope is very slow as $y \rightarrow+\infty$ also, especially for the Airy function when $k=1$, and is faster for $k=2$ in Figure 3 ,

For convenience we denote these rescaled kernels as higher-order Airy functions by

$$
F(y)=\mathrm{Ai}_{2 k+1}(y) \quad \text { for } \quad k=1,2, \ldots, \quad \text { so that } \mathrm{Ai}=\mathrm{Ai}_{3} .
$$

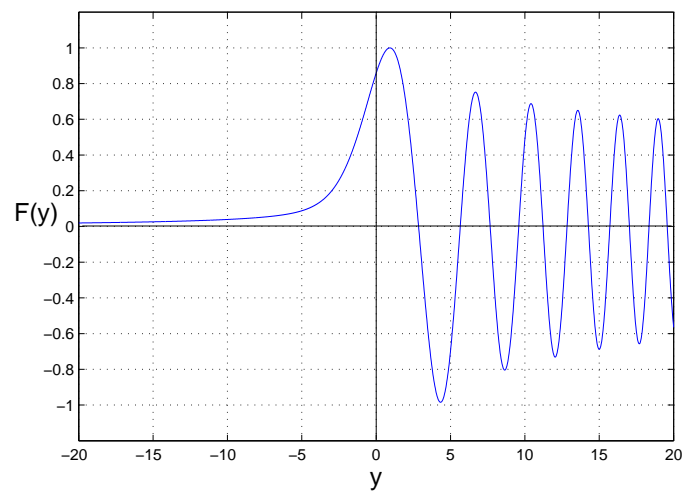

(a) Oscillations of $F(y)$ for $y \in(0,20)$.

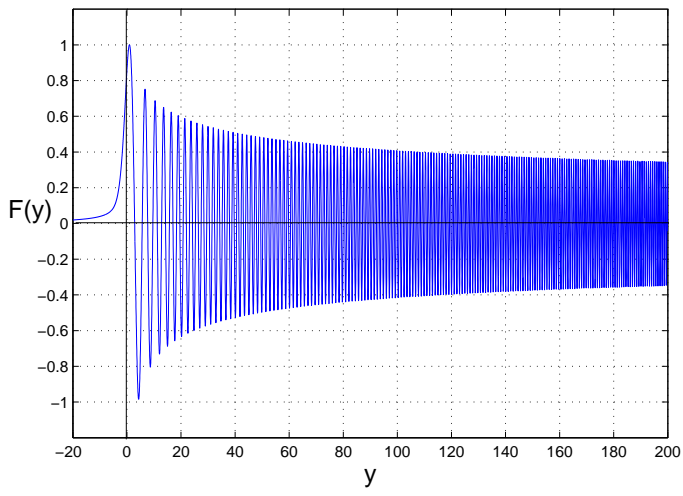

(b) Oscillatory "tail" of $F(y)$ for $y \gg 1$.

FiguRE 1. The rescaled kernel $F(y)=\operatorname{Ai}(y)$ of the fundamental solution (2.1) of (1.5) $(k=1)$ obtained by two-sided shooting.

\section{Explicit SEMigRoup REPRESENTATION: FIRST SPECTRAL PROPERTIES}

Here, we begin to develop first aspects of a Hermitian spectral theory, which our further analysis will depend crucially on.

3.1. Operator B: formal series. Let $u(x, t)$ be the solution of the Cauchy problem for the LDE- $(2 k+1)$ (1.19), with bounded measurable initial data $u(x, 0)=u_{0}(x)$ from a weighted $L^{2}$ space to be specified. The solution is then represented by the convolution of 


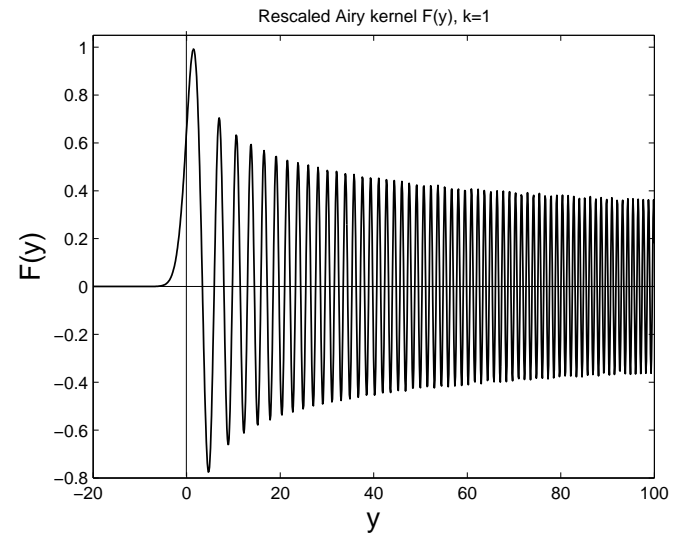

FigurE 2. The rescaled kernel $F(y)=\operatorname{Ai}(y)$ of the ODE (2.4) $(k=1)$ obtained by one-sided shooting via the bundle (2.10).

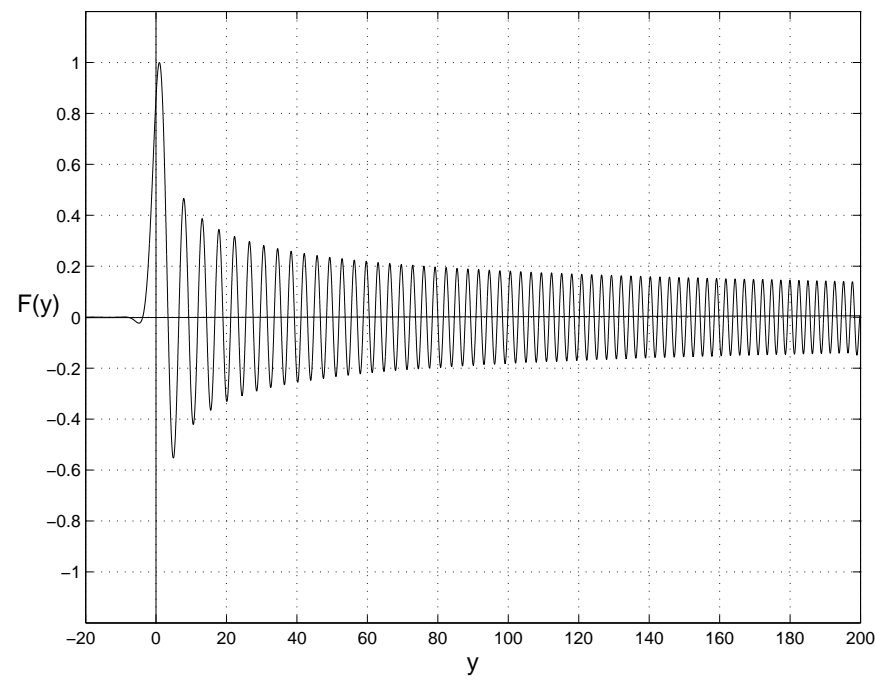

FiguRE 3. The rescaled kernel $F(y)=\mathrm{Ai}_{5}(y)$ of the fundamental solution (2.1) of the LDE-5 $u_{t}=-u_{x x x x x}(k=2)$ obtained by two-sided shooting.

initial data with the fundamental solution given by a Poisson-type integral:

$$
u(x, t)=b(t) * u_{0} \equiv t^{-\frac{1}{2 k+1}} \int_{\mathbb{R}} F\left((x-z) t^{-\frac{1}{2 k+1}}\right) u_{0}(z) \mathrm{d} z .
$$

Let us next introduce further rescaling corresponding to the variables of the fundamental solution (2.1),

$$
u(x, t)=t^{-\frac{1}{2 k+1}} w(y, \tau), \quad \text { where } \quad y=x / t^{\frac{1}{2 k+1}}, \quad \tau=\ln t: \mathbb{R}_{+} \rightarrow \mathbb{R},
$$


where we now have scaling with respect to time as well. The rescaled solution $w(y, \tau)$ then satisfies the evolution equation

$$
w_{\tau}=\mathbf{B} w
$$

where $\mathbf{B}$ is the linear operator for the rescaled kernel described in (2.2). More precisely, $\mathbf{B}$ is still a formal linear differential expression to be equipped with proper "boundary conditions" to get its actual domain.

Here, $w(y, \tau)$ satisfies the Cauchy problem for $(3.3)$ in $\mathbb{R} \times \mathbb{R}_{+}$, with initial data at $\tau=0$, i.e., at $t=1$ and not $t=0$. So now the initial data are given by

$$
w_{0}(y)=u(y, 1) \equiv b(1) * u_{0}=F * u_{0} .
$$

Hence, the linear operator $\frac{\partial}{\partial \tau}-\mathbf{B}$ is the rescaled version of the original linear dispersion operator $\frac{\partial}{\partial t}+(-1)^{k} D_{x}^{2 k+1}$. Therefore, the corresponding semigroup $\mathrm{e}^{\mathbf{B} \tau}$ admits an explicit integral representation. This helps to establish some properties of $\mathbf{B}$, including key spectral ones, and describe other evolution features of the linear flow.

Thus, rescaling convolution (3.1) gives the explicit representation of the semigroup

$$
w(y, \tau)=\int_{\mathbb{R}} F\left(y-z \mathrm{e}^{-\frac{\tau}{2 k+1}}\right) u_{0}(z) \mathrm{d} z \equiv \mathrm{e}^{\mathbf{B} \tau} u(y, 1) \quad \text { for } \quad \tau \geq 0 .
$$

For any $y \in \mathbb{R}$, Taylor's power series for the analytic kernel $F$ can be used to expand the convolution and to obtain

$$
F\left(y-z \mathrm{e}^{-\frac{\tau}{2 k+1}}\right)=\sum_{(\beta)} \mathrm{e}^{-\frac{|\beta| \tau}{2 k+1} \frac{(-1)^{|\beta|}}{\beta !}} D_{y}^{\beta} F(y) z^{\beta} \equiv \sum_{(\beta)} \mathrm{e}^{-\frac{|\beta| \tau}{2 k+1}} \frac{1}{\sqrt{\beta !}} \psi_{\beta}(y) z^{\beta} .
$$

Here, for the first time, we reveal the eigenvalues and eigenfunctions of the operator $\mathbf{B}$ as

$$
\lambda_{\beta}=-\frac{|\beta|}{2 k+1}^{2 k} \quad \text { and } \quad \psi_{\beta}(y)=\frac{(-1)}{\sqrt{\beta !}}^{|\beta|} D_{y}^{\beta} F(y) \text { for any (multiindex) } \beta, \quad|\beta| \geq 0 \text {. }
$$

Remark: on extensions to $\mathbb{R}^{N}$. We note that whilst $|\beta| \equiv \beta$ here, this is not the case in the multi-dimensional $\mathbb{R}^{N}$, where $\beta$ stands for a multiindex. However, we often still keep the notation of $|\beta|$, to show that the theory here can be extended to multi-dimensional spaces. We bear in mind that Hermitian spectral theory can be properly developed for the typical linear dispersion operator in $\mathbb{R}^{N}$,

$$
\mathbf{B}=(-1)^{k+1} \frac{\partial}{\partial y_{1}} \Delta^{k}+\frac{1}{2 k+1} y \cdot \nabla_{y}+\frac{N}{2 k+1} I,
$$

which appears after the scaling (similar to (3.2)) of the following LDE:

$$
u_{t}=(-1)^{k} \frac{\partial}{\partial x_{1}} \Delta^{k} u,
$$

where analogies of the operator on the right-hand side occur in completely integrable equation theory (cf. the Kadomtsev-Petviashvili equation for $N=2$ and others from higher-order hierarchies). The multi-index is then $\beta=\left(\beta_{1}, \ldots, \beta_{N}\right)$, with the length $|\beta|=\beta_{1}+\ldots+\beta_{N}$. With this notation, some of our basic results can be directly translated to operators such as (3.8) in $\mathbb{R}^{N}$; see [13, 18] for necessary technical details. 
Thus, without loss of generality, we continue to develop a 1D theory. Looking back at the expansion of the convolution (3.5) via (3.6), the solution of (3.3) is given by the series (understood formally still):

$$
w(y, \tau)=\sum_{(\beta)} \mathrm{e}^{-\frac{\beta \tau}{2 k+1}} M_{\beta}\left(u_{0}\right) \psi_{\beta}(y)
$$

It then follows that $\lambda_{\beta}=-\frac{\beta}{2 k+1}$ and $\psi_{\beta}(y)$ are the eigenvalues and eigenfunctions of $\mathbf{B}$ as in (3.7) and

$$
M_{\beta}\left(u_{0}\right)=\frac{1}{\sqrt{\beta !}} \int_{\mathbb{R}} z^{\beta} u_{0}(z) \mathrm{d} z,
$$

then turns out to be corresponding moments of the initial data $w_{0}$, i.e., "scalar products" of $w_{0}$ with some "adjoint polynomials" (eigenfunctions of the "adjoint" operator $\mathbf{B}^{*}$ ) to be detected shortly together with a proper metric involved.

3.2. B: convergence of series. We next need to check suitable metrics of convergence of formal series (3.10). Those questions were addressed in [13] and in the most general case in [18] in the purely "elliptic" ("parabolic") case, when the rescaled kernel $F(y)$ always have exponential decay as $y \rightarrow \infty$. In view of (2.7), this is not currently the case as $y \rightarrow+\infty$. We concentrate on this case and refer to the analysis in [18, $\S 5]$ of convergence of the integrals as $y \rightarrow-\infty$.

First of all, as in [13, 18], we have that the ODE (2.2) for $F$ implies the following estimate of the eigenfunctions:

$$
\left|\psi_{\beta}(y)\right| \leq \frac{c}{\sqrt{\beta !}}(1+y)^{\frac{\beta}{2 k}-\frac{2 k-1}{4 k}} \alpha_{k}^{\beta} \quad \text { for } \quad y \geq 0, \quad \text { where } \quad \alpha_{k}=\frac{2 k+1}{2 k} d_{k} \sin b_{k} .
$$

Here, the main growing factor $\sim y^{\frac{\beta}{2 k}}$ as $y \rightarrow+\infty$, as well as the multiplier $\alpha_{k}^{\beta}$, come from differentiating $\beta$ times inside the cos function in (2.7).

We next claim that the series in (3.10) converges uniformly on compact subsets for data from the weighted $L^{2}$ space (e.g., with fast exponential decay at infinity):

$$
u_{0} \in L_{\hat{\rho}}^{2}(\mathbb{R}), \quad \text { where } \quad \hat{\rho}(y)=\mathrm{e}^{a|y|^{\alpha}}, \quad \alpha=\frac{2 k+1}{2 k},
$$

and $a>0$ is a sufficiently small constant. As in the parabolic case [13], in view of the exponential decay (2.8), for $y<0$ the condition $a \in\left(0,2 \hat{d}_{k}\right)$ is necessary, but this case will not be treated here as being standard.

The proof of uniform convergence on compact subsets uses typical Hölder estimates such as (for $y>0$ again)

$$
\int|z|^{\beta}\left|u_{0}\right| \equiv \int \frac{1}{\sqrt{\hat{\rho}}}|z|^{\beta} \sqrt{\hat{\rho}}\left|u_{0}\right| \leq \sqrt{\int \hat{\rho}\left|u_{0}\right|^{2}} \sqrt{\int \frac{1}{\hat{\rho}}|z|^{2 \beta}}
$$

and, using standard properties of the $\Gamma$-function and Stirling's formula, we estimate the last integral as follows:

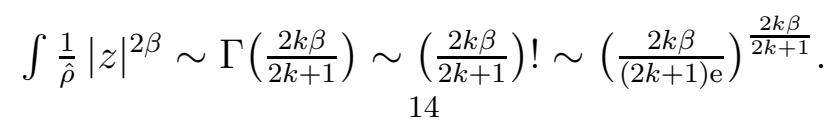


Overall, this gives the following majorizing series for (3.10) for $y \in(0, L)$ :

$$
\sum_{(\beta)} \frac{1}{\sqrt{\beta !}} \sqrt{\left(\frac{2 k \beta}{2 k+1}\right) !} L^{\frac{\beta}{2 k}},
$$

which obviously converges for arbitrary fixed $L>0$.

We next show that the series (3.10) converges in the mean in the metric of the weighted space

$$
L_{\rho}^{2}(\mathbb{R}), \quad \text { where } \quad \rho(y)= \begin{cases}\mathrm{e}^{a|y|^{\alpha}} & \text { for } y \leq-1, \\ \mathrm{e}^{-a y^{\alpha}} & \text { for } y \geq 1,\end{cases}
$$

cf. (3.13). Using the same estimates (3.12) and (3.14), we then derive the following more sensitive geometric majorizing series:

$$
\sum_{(\beta)} \gamma_{k}^{\frac{\beta}{2 k+1}}, \quad \text { where } \quad \gamma_{k}=\frac{1}{2 k+1}\left(\frac{2 k}{2 k+1}\right)^{2 k-1}<1,
$$

so the series converges. We do not include in $\gamma_{k}$ the extra multipliers $\sim\left|d_{k} \sin b_{k}\right|<1$ shown in (3.12), since the first one $\frac{2 k}{2 k+1}<1$ already suffices for the convergence of (3.18).

3.3. B: alternative representation of semigroup and series, generalized Hermite polynomials. Let us discuss an equivalent explicit representation of the semigroup for $\mathbf{B}$, which, though being more difficult, will more clearly determine the eigenfunctions of the adjoint operator $\mathbf{B}^{*}$ to be introduced and studied next by a simpler direct approach. We perform another rescaling to exclude the relation (3.4), in order to find the correct semigroup, corresponding to the initial data at $t=0$, i.e., $w_{0}=u_{0}$ :

$$
u=(1+t)^{-\frac{1}{2 k+1}} w, \quad y=x(1+t)^{-\frac{1}{2 k+1}}, \quad \tau=\ln (1+t): \mathbb{R}_{+} \rightarrow \mathbb{R}_{+} .
$$

Then rescaling the convolution gives

$$
w(y, \tau)=\mathrm{e}^{\mathbf{B} \tau} u_{0} \equiv\left(1-\mathrm{e}^{-\tau}\right)^{-\frac{1}{2 k+1}} \int_{\mathbb{R}} F\left(\left(y-z \mathrm{e}^{-\frac{\tau}{2 k+1}}\right)\left(1-\mathrm{e}^{-\tau}\right)^{-\frac{1}{2 k+1}}\right) w_{0}(z) \mathrm{d} z .
$$

Once again we can look to find explicit representations for the eigenfunctions, eigenvalues, and adjoint eigenfunctions, given in the dual products $\left\langle u_{0}, \psi_{\beta}^{*}\right\rangle$, in the standard metric of $L^{2}$ (actually, a more delicate indefinite metric will be needed; see below). It will be shown later that we can actually determine the adjoint eigenfunctions, $\left\{\psi_{\beta}^{*}\right\}$, using a much easier method.

Meantime, let us perform some convenient manipulations. Looking at our rescaled equation (3.19), by Taylor's expansion we have

$$
\begin{gathered}
F\left(\left(y-z \mathrm{e}^{-\frac{\tau}{2 k+1}}\right)\left(1-\mathrm{e}^{-\tau}\right)^{-\frac{1}{2 k+1}}\right)=\sum_{(\mu)} \frac{(-1)}{\mu !}^{\mu} D_{y}^{\mu} F\left(y\left(1-\mathrm{e}^{-\tau}\right)^{-\frac{1}{2 k+1}}\right)\left(\mathrm{e}^{\tau}-1\right)^{-\frac{\mu}{2 k+1}} z^{\mu} \\
\text { and } F\left(y\left(1-\mathrm{e}^{-\tau}\right)^{-\frac{1}{2 k+1}}\right)=\sum_{(\nu)} \frac{1}{\nu !}\left(D_{y}^{\nu} F\right)(0) y^{\nu}\left(1-\mathrm{e}^{-\tau}\right)^{-\frac{\nu}{2 k+1}} .
\end{gathered}
$$

Then, using these expansions, our solution is given by

$$
\begin{gathered}
w(y, \tau)=\left(1-\mathrm{e}^{-\tau}\right)^{-\frac{1}{2 k+1}} \sum_{(\mu, \nu)} \frac{(-1)^{\mu}}{\mu ! \nu !}\left(D_{y}^{\nu} F\right)(0) D_{y}^{\mu}\left(y^{\nu}\right)\left(\mathrm{e}^{\tau}-1\right)^{-\frac{\mu}{2 k+1}} \\
\times\left(1-\mathrm{e}^{-\tau}\right)^{-\frac{\nu}{2 k+1}} \int_{\mathbb{R}} z^{\mu} w_{0}(z) \mathrm{d} z . \\
1{ }^{2}
\end{gathered}
$$


Rearranging this, we have

$$
w(y, \tau)=\sum_{(\mu, \nu)} \mathrm{e}^{-\frac{\mu \tau}{2 k+1}}\left(1-\mathrm{e}^{-\tau}\right)^{-\frac{\mu+\nu+1}{2 k+1}} \frac{(-1)^{\mu}}{\mu ! \nu !}\left(D_{y}^{\nu} F\right)(0) D_{y}^{\mu}\left(y^{\nu}\right) \int_{\mathbb{R}} z^{\mu} w_{0}(z) \mathrm{d} z .
$$

Further expansion in the exponential term containing $\left(1-\mathrm{e}^{-\tau}\right)^{-\frac{\mu+\nu+1}{2 k+1}}$ in terms of $\mathrm{e}^{-\tau}$ yields an alternative more complete eigenfunction expansion of the semigroup with a full representation of all the eigenfunctions and the generalized Hermite polynomials $\left\{\psi_{\beta}^{*}\right\}$ in the moments $M_{\beta}$. Moreover, being re-written in the form

$$
w(y, \tau)=\sum_{(\beta)} \mathrm{e}^{-\frac{\beta \tau}{2 k+1}} \psi_{\beta}(y)\left\langle w_{0}, \psi_{\beta}^{*}\right\rangle_{*},
$$

this expansion, besides the adjoint polynomials $\Phi^{*}=\left\{\psi_{\beta}^{*}\right\}$, also defines the corresponding indefinite metric $\langle\cdot, \cdot\rangle_{*}$ to be carefully introduced and explained in Section 5, where those polynomials will be obtained via a simpler direct approach.

3.4. Classification of large-time asymptotics. As a by-product, for our LDEs, we have described the large-time asymptotic behaviour for the problem

$$
u_{t}=(-1)^{k+1} D_{x}^{2 k+1} u \quad \text { in } \quad \mathbb{R} \times \mathbb{R}_{+}, \quad \text { where } \quad u_{0} \in L_{\hat{\rho}}^{2},
$$

and the weight $\hat{\rho}$ is as in (3.13).

Theorem 3.1. For the problem (3.23), the eigenfunction expansion (3.10) implies that, for any $u_{0} \in L_{\hat{\rho}}^{2}(\mathbb{R}), u_{0} \neq 0$, there exists a finite $l$ such that, as $t \rightarrow+\infty$,

$$
u(x, t)=t^{-\frac{1}{2 k+1}+\lambda_{\beta}}\left[c_{\beta} \psi_{\beta}\left(x / t^{\frac{1}{2 k+1}}\right)+o(1)\right], \text { where } \lambda_{\beta}=-\frac{l}{2 k+1}
$$

and $l=|\beta|$ is the first eigenvalue index, with the corresponding moment $c_{\beta}=M_{\beta}\left(u_{0}\right) \neq 0$.

The following uniqueness conclusion is straightforward and we keep this as a simple illustration for further results:

Corollary 3.1. Assume that, for any constant $K>0$, the solution of (3.23) satisfies

$$
\sup _{x}|u(x, t)|=o\left(t^{-K}\right) \quad \text { as } t \rightarrow \infty .
$$

Then $u(x, t) \equiv 0$.

Such results belong to classic Carleman-Agmon-type estimates in operator theory: if a solution of a linear equation, under proper conditions on operators involved, decays super-exponentially fast (in terms of $\tau=\ln t$ ) as $t \rightarrow+\infty$, then it is trivial. For elliptic equations $P(x, D) u=0$, this has the natural counterpart on strong unique continuation property saying that nontrivial solutions cannot have zeros of infinite order; a result first proved by Carleman in 1939 for $P=-\Delta+V, V \in L_{\mathrm{loc}}^{\infty}$, in $\mathbb{R}^{2}$ [8]; see [30, 16, 55] for further references and modern extensions.

Thus, for convenience, we fix again some conclusions achieved above:

- There exists point spectrum $\left\{\lambda_{\beta}=-\frac{\beta}{2 k+1}, \beta \geq 0\right\}$ of non-symmetric operator $\mathbf{B}$.

- We have no integral terms in the expansion, hence the spectrum is expected to be discrete in a proper functional setting. 
- The set of eigenfunctions $\left\{\psi_{\beta}\right\}$ seems to be complete and closed in a certain weighted $L^{2}$-space.

- Traces of the polynomials, which give rise to the adjoint eigenfunctions, $\left\{\psi_{\beta}^{*}\right\}$.

3.5. Semigroup of the adjoint operator $\mathbf{B}^{*}$ and its eigenfunction expansion. We now find the explicit representation of the semigroup $\mathrm{e}^{\mathbf{B}^{*} \tau}$, where $\mathbf{B}^{*}$ is obtained from the LDE in (3.23) by using other blow-up rescaling. Let us introduce the rescaled variables

$$
u(x, t)=w(y, \tau), \quad y=x /(1-t)^{\frac{1}{2 k+1}}, \quad \tau=-\ln (1-t):(0,1) \rightarrow \mathbb{R}_{+},
$$

where, for convenience, the blow-up time is $T=1$. Then $w$ now solves the problem

$$
w_{\tau}=\mathbf{B}^{*} w \quad \text { for } \quad \tau>0
$$

with data $w(y, 0)=w_{0}(y) \equiv u_{0}(y)$. Here the "adjoint" operator $\mathbf{B}^{*}$ is given by

$$
\mathbf{B}^{*}=(-1)^{k+1} D_{y}^{2 k+1}-\frac{1}{2 k+1} y D_{y} .
$$

By rescaling the convolution (3.1), we have

$$
w(y, \tau)=\left(1-\mathrm{e}^{-\tau}\right)^{-\frac{1}{2 k+1}} \int_{\mathbb{R}} F\left(\left(y \mathrm{e}^{-\frac{\tau}{2 k+1}}-z\right)\left(1-\mathrm{e}^{-\tau}\right)^{-\frac{1}{2 k+1}}\right) w_{0}(z) \mathrm{d} z .
$$

Using Taylor's expansion yields

$$
\begin{aligned}
& F\left(\left(y \mathrm{e}^{-\frac{\tau}{2 k+1}}-z\right)\left(1-\mathrm{e}^{-\tau}\right)^{-\frac{1}{2 k+1}}\right) \\
= & \sum_{(\beta)} \frac{(-1)^{\beta}}{\beta !}\left(1-\mathrm{e}^{-\tau}\right)^{-\frac{\beta}{2 k+1}} D^{\beta} F\left(y \mathrm{e}^{-\frac{\tau}{2 k+1}}\left(1-\mathrm{e}^{-\tau}\right)^{-\frac{1}{2 k+1}}\right) z^{\beta}
\end{aligned}
$$

and expanding in $y$ leads to

$$
F\left(y \mathrm{e}^{-\frac{\tau}{2 k+1}}\left(1-\mathrm{e}^{-\tau}\right)^{-\frac{1}{2 k+1}}\right)=\sum_{(\nu)} \frac{1}{\nu !} D^{\nu} F(0) y^{\nu}\left(\mathrm{e}^{\tau}-1\right)^{-\frac{\nu}{2 k+1}} .
$$

So the solution is represented as

$$
\begin{gathered}
w(y, \tau)=\left(1-\mathrm{e}^{-\tau}\right)^{-\frac{1}{2 k+1}} \sum_{(\beta, \nu)} \frac{(-1)^{\beta}}{\beta ! \nu !}\left(1-\mathrm{e}^{-\tau}\right)^{-\frac{\beta}{2 k+1}}\left(\mathrm{e}^{\tau}-1\right)^{-\frac{\nu}{2 k+1}} \\
\times D^{\nu} F(0) \frac{1}{(\nu-\beta) !} y^{\nu-\mu} \int_{\mathbb{R}} z^{\beta} w_{0}(z) \mathrm{d} z
\end{gathered}
$$

Again, similar to the expansion (3.22) for the operator B, (3.26) can be viewed as an eigenfunction expansion of the solution that can reveal many key spectral properties of $\mathbf{B}^{*}$. In particular, we obtain the same real spectrum and eigenfunctions:

$$
\mathbf{B}^{*}: \quad \lambda_{\beta}=-\frac{\beta}{2 k+1} \text { and Hermite-type polynomial eigenfunctions } \psi_{\beta}^{*}(y) .
$$

However, further refining of this expansion will lead to more complicated formulae, which are not that effective and useful. Therefore, we return later on to polynomial eigenfunctions of $\mathbf{B}^{*}$ on the basis of a simpler direct approach. 
3.6. Classification of multiple zeros: micro-scale blow-up asymptotics. Due to the blow-up character of the scaling (3.24), the polynomial eigenfunction set $\Phi^{*}=\left\{\psi_{\beta}^{*}\right\}$ of the adjoint operator $\mathbf{B}^{*}$ is able to give insight into the structure of multiple spatial zeros of solutions $u(x, t)$ of the corresponding LDEs. This blow-up asymptotic theory is a natural counterpart of the large-time one in Section 3.4 induced by eigenfunctions $\Phi$ of B. The key principles of such a classification for LDEs can be found in [18, $\S 9$ ], where other blow-up application of such a spectral analysis can be found.

\section{Hermitian Spectral theory: operator B}

We now start more systematically to develop necessary spectral theory for the operator pair $\left\{\mathbf{B}, \mathbf{B}^{*}\right\}$ introduced above. In some aspects, this theory repeats standard steps of selfadjoint theory for the Hermite classic operator (1.10), which since the nineteenth century, is associated with the names of Sturm, Hermite, and other famous mathematicians.

4.1. Bounded operator B. According to (3.17) and (3.13), we calculate the spectrum of the linear operator $\mathbf{B}$ in the weighted space $L_{\rho}^{2}(\mathbb{R})$, with an exponential weight given in (3.17), where, as usual, we assume $\rho(y)$ to be sufficiently smooth in the complement interval $[1,-1]$. Here we have that $\rho(y)>0$ and $a \in\left(0,2 \hat{d}_{k}\right)$ (this is necessary for $y \ll-1$ only) is a sufficiently small positive constant, where $\hat{d}_{k}$ is defined as before, in (2.7), (2.9).

We introduce a Hilbert space of functions $H_{\rho}^{2 k+1}(\mathbb{R})$ with the inner product and the induced norms:

$$
\langle v, w\rangle_{2 k+1, \rho}=\int_{\mathbb{R}} \rho(y) \sum_{r=0}^{2 k+1} D_{y}^{r} v(y) D_{y}^{r} w(y) \mathrm{d} y, \quad\|v\|_{2 k+1, \rho}^{2}=\int_{\mathbb{R}} \rho(y) \sum_{r=0}^{2 k+1}\left|D_{y}^{r} v(y)\right|^{2} \mathrm{~d} y .
$$

It turns out that it suffices to restrict to the real case. Then $H_{\rho}^{2 k+1} \subset L_{\rho}^{2}(\mathbb{R}) \subset L^{2}(\mathbb{R})$. The first conclusion is standard as in [13]:

Lemma 4.1. B is a bounded linear operator from $H_{\rho}^{2 k+1}(\mathbb{R})$ to $L_{\rho}^{2}(\mathbb{R})$.

Proof. Consider the differential expression (2.2),

$$
\mathbf{B} v=(-1)^{k+1} v^{(2 k+1)}+\frac{1}{2 k+1} y v^{\prime}+\frac{1}{2 k+1} v .
$$

For $\mathbf{B}$ to be bounded, it is necessary to look at the second term with the unbounded coefficient $y$. In order to do this, we want to show that

$$
\int \rho\left(y v^{\prime}\right)^{2} \leq C \int \rho\left(v^{(2 k+1)}\right)^{2} \mathrm{~d} y,
$$

for some constant $C>0$. To show this, we look at the non-negative integral

$$
0 \leq \int \rho\left(v^{\prime}+y^{\gamma} v\right)^{2} \mathrm{~d} y=\int \rho\left(\left(v^{\prime}\right)^{2}+y^{2 \gamma} v^{2}+2 y^{\gamma} v^{\prime} v\right) \mathrm{d} y
$$

where $\gamma>0$ is some unknown exponent. Integrating by parts (allowed for functions in $\left.H_{\rho}^{2 k+1}(\mathbb{R})\right)$ in the last term yields

$$
2 \int\left(\rho y^{\gamma}\right) v^{\prime} v \mathrm{~d} y=\int\left(\rho y^{\gamma}\right)\left(v^{2}\right)^{\prime} \mathrm{d} y=-\int v^{2}\left(\rho y^{\gamma}\right)^{\prime} \mathrm{d} y
$$


Then for the exponential weight, we have to have that

$$
\rho(y)=\mathrm{e}^{-a y^{\alpha}} \quad \Longrightarrow \quad\left(\rho y^{\gamma}\right)^{\prime} \sim \mathrm{e}^{-a y^{\alpha}} y^{\gamma+\alpha-1},
$$

for $y \gg 1$ (and also for $y \ll-1$ that is similar, where we replace $y \mapsto|y|$ ). Hence

$$
\int \rho\left(v^{\prime}\right)^{2} \mathrm{~d} y+\int \rho y^{2 \gamma} v^{2} \mathrm{~d} y+C_{1} \int \rho y^{\gamma+\alpha-1} v^{2} \mathrm{~d} y \geq 0 .
$$

By equating powers of $y$, this yields $\gamma=\alpha-1$. Substituting this $\gamma$, we have that

$$
\int \rho y^{2(\alpha-1)} v^{2} \mathrm{~d} y \leq C_{2} \int \rho\left(v^{\prime}\right)^{2} \mathrm{~d} y \text {. }
$$

In particular, for $k=1$, by the Hardy-type inequality, the following holds:

$$
\int \rho y^{4(\alpha-1)}\left(v^{\prime}\right)^{2} \mathrm{~d} y \leq C_{3} \int \rho y^{2(\alpha-1)}\left(v^{\prime \prime}\right)^{2} \mathrm{~d} y \leq C_{3}^{2} \int \rho\left(v^{\prime \prime \prime}\right)^{2} \mathrm{~d} y .
$$

However, we want that

$$
\int \rho y^{2}\left(F^{\prime}\right)^{2} \mathrm{~d} y \leq C_{4} \int \rho\left(F^{\prime \prime \prime}\right)^{2} \mathrm{~d} y .
$$

Hence we have $4(\alpha-1)=2$, which gives $\alpha=\frac{3}{2}$.

For the general case, we have $2 k$ iterations and so

$$
4 k(\alpha-1)=2 \quad \Longrightarrow \quad \alpha=\frac{2 k+1}{2 k} \text {. }
$$

4.2. Completeness and compact resolvent. The following conclusions are also pretty general [13, 18]:

Lemma 4.2. (i) The set of eigenfunctions $\Phi=\left\{\psi_{\beta}\right\}$ given in (3.7) is complete in $L^{2}(\mathbb{R})$ and in $L_{\hat{\rho}}^{2}(\mathbb{R})$.

(ii) The resolvent $(\mathbf{B}-\lambda I)^{-1}$ is a compact operator in $L_{\hat{\rho}}^{2}(\mathbb{R})$.

Proof. (i) In fact, due to the differential structure of the eigenfunctions in (3.7), this is a standard result in functional analysis; see analytic function theory approach in Kolmogorov-Fomin [38, p. 431]. Let us show that the system of eigenfunctions $\left\{D^{\beta} F\right\}$ is complete in $L_{\hat{\rho}}^{2}(\mathbb{R})$; the case of $L^{2}(\mathbb{R})$ is simpler. By the Riesz-Fischer theorem, we have to show that, given a function $G \in L_{\hat{\rho}}^{2}(\mathbb{R})$, then

$$
\int D^{\beta} F(x) G(x) \mathrm{d} x=0 \text { for any } \beta,
$$

implies that $G=0$. Let $\hat{F}=\mathcal{F}(F)$ and $\hat{G} \mathcal{F}(G)$ be the Fourier transforms of $F$ and $G$. Then substituting $F=\mathcal{F}^{-1}(\hat{F}), G=\mathcal{F}^{-1}(\hat{G})$, and using that $D^{\beta} \mathcal{F}^{-1}(\hat{F}) \sim \xi^{\beta} \mathcal{F}^{-1}(\hat{F})$, after integration by parts via $\mathcal{F}^{-1}(\hat{G})(\xi) \sim \mathcal{F}(\hat{G})(-\xi)$, we obtain that

$$
\int \xi^{\beta} \hat{F}(\xi) \hat{G}(-\xi) \mathrm{d} \xi=0 \quad \text { for any } \quad \beta .
$$

Let us calculate $\hat{F}$. Applying the Fourier transform to the equation (2.2) yields

$$
\mathrm{i}|\xi|^{2 k} \xi \hat{F}+\frac{1}{2 k+1} \xi D \hat{F}=0 \quad \Longrightarrow \quad \hat{F}(\xi)=\mathrm{e}^{-\mathrm{i}|\xi|^{2 k} \xi} \text {. }
$$

Substituting (4.2) into (4.1) yields

$$
\int \xi^{\beta} \mathrm{e}^{-\mathrm{i}|\xi|^{2 k} \xi} \hat{G}(-\xi) \mathrm{d} \xi=0 \text { for any } \beta .
$$


Finally, consider the function

$$
M(z)=\int \mathrm{e}^{-\mathrm{i}|\xi|^{2 k} \xi} \hat{G}(-\xi) \mathrm{e}^{\mathrm{i} z \xi} \mathrm{d} \xi,
$$

which is entirely analytic in $\mathbb{C}$, [37]. So (4.3) means that $D^{\beta} M(0)=0$ for any $\beta$. Therefore, $M(z) \equiv 0$. Hence $\mathcal{G}(\xi)=0$ almost everywhere and $G=0$.

(ii) The proof follows that of the $2 m$ th-order case, set out in [13, $\S 2.3]$. In fact, replacing $m \mapsto k+\frac{1}{2}$, will yield the same result. This also directly follows from the compact embedding of the corresponding spaces $H_{\rho}^{2 k+1} \subset L_{\rho}^{2}$; see Maz'ya's classic monograph on Sobolev spaces [44, p. 40].

4.3. Space of closure $\tilde{L}_{\rho}^{2}(\mathbb{R})$. We next apply another direct approach to constructing the necessary domain of $\mathbf{B}$; cf. that in [13]. Such a rather "artificial" construction, though not being completely unavoidable for such odd-order operator $\mathbf{B}$ (a more traditional way of doing this along the lines in [13, 18] is still available at least partially), will essentially simplify in Section 5 the way of introducing the set of linear functionals $\Phi^{*}=\left\{\psi_{\beta}^{*}\right\}$ from the adjoint space being generalized Hermite polynomials as eigenfunctions of the adjoint operator $\mathbf{B}^{*}$.

Namely, as in [18, $\S 5]$, given the complete set of eigenfunctions $\Phi=\left\{\psi_{\beta}\right\}$ we introduce the space of $\Phi$-closure denoted by $\tilde{L}_{\rho}^{2}(\mathbb{R}) \subset L_{\rho}^{2}(\mathbb{R})$ as follows:

$$
\tilde{L}_{\rho}^{2}(\mathbb{R}): \quad \text { closure of finite sums } \sum_{\beta=0}^{M} c_{\beta} \psi_{\beta} \text { in the metric of } L_{\rho}^{2}(\mathbb{R}) .
$$

The notation the "space of closure" is then justified naturally:

(4.5) any $v \in \tilde{L}_{\rho}^{2}(\mathbb{R})$ has the unique eigenfunction expansion via $\Phi: \quad v=\sum_{(\beta \geq 0)} c_{\beta} \psi_{\beta}$,

i.e., the set $\Phi$ is closed therein. Note that, for the whole space $L_{\rho}^{2}(\mathbb{R})$, the property (4.5) is not easy to check and, most plausibly, it fails. A functional characterization of such a space is not that straightforward, and some estimates of the sequences $\left\{c_{\beta}\right\}$, for which the series in (4.5) converges, can be derived as in [18, $\S 5]$, so we will not treat those questions here. In Appendix A, we present an alternative explanation of some of key "radiation condition" properties for functions from the space of $\Phi$-closure. Appendix B shows spectral theory for a majorizing integral operator for (3.1), which is order-preserving so some comparison features are available.

We now complete our spectral theory of $\mathbf{B}$ as follows:

Lemma 4.3. The operator $\mathbf{B}$ in (2.2) with the domain $\tilde{\mathcal{D}}(\mathbf{B})=\tilde{L}_{\rho}^{2}(\mathbb{R}) \cap H_{\rho}^{2 k+1}(\mathbb{R})$ has the point spectrum comprising real eigenvalues only:

$$
\sigma(\mathbf{B})=\left\{\lambda_{\beta}=-\frac{\beta}{2 k+1}, \beta=0,1,2, \ldots\right\} .
$$

Eigenvalues are simple with eigenfunctions (3.7).

Proof. Eigenvalues and eigenfunctions are found by applying $D_{y}^{\beta}$ to (2.2):

$$
D_{y}^{\beta} \mathbf{B} F \equiv \mathbf{B} D_{y}^{\beta} F+\frac{\beta}{2 k+1} D_{y}^{\beta} F=0 .
$$

By the construction of the space of closure (4.4), this completes the proof. 
Note also that as follows from the asymptotic expansion of (3.10) and (3.20) as $\tau \rightarrow$ $\infty$, no other eigenfunctions exist (at least for such data). This again confirms that all eigenvalues are real and are given in (4.6).

A sharper using Stirling's series shows that (see estimates in [18, § 5.1] that are applied to the present case)

$$
v=\sum c_{\beta} \psi_{\beta} \in \tilde{L}_{\rho}^{2} \Longrightarrow c_{\beta}=o\left(|\beta|^{|\beta|(\nu+\varepsilon)}\right) \quad \text { with an } \varepsilon>0,
$$

for "almost all" $|\beta| \gg 1$. A sharper and almost optimal estimate reads:

$$
\sum c_{\beta} \psi_{\beta} \in \tilde{L}_{\rho}^{2} \quad \text { if } \quad c_{\beta}=O\left(\delta^{|\beta|}|\beta|^{|\beta| \nu}\right), \quad \text { where } \quad \nu=\frac{2-\alpha}{2 \alpha}=\frac{2 k-1}{2(2 k+1)},
$$

with a sufficiently small constant $\delta>0$.

By $\tilde{H}_{\rho}^{2 k+1} \subset \tilde{L}_{\rho}^{2}$, we denote the dense linear subspace obtained as the closure in the norm of $H_{\rho}^{2 k+1}$ of the subset of eigenfunction expansions with coefficients satisfying (4.7) or (4.8). $\tilde{H}_{\rho}^{2 k+1}$ with the scalar product of $H_{\rho}^{2 k+1}$ becomes a Hilbert space and can be considered as the domain of $\mathbf{B}$ in $H_{\rho}^{2 k+1}$. There holds

$$
\tilde{H}_{\rho}^{2 k+1} \subseteq H_{\rho}^{2 k+1} \cap \tilde{L}_{\rho}^{2} .
$$

4.4. Little Hilbert spaces. We will need a subspace of $\tilde{L}_{\rho}^{2}$ introduced as a little Hilbert space $l_{\rho}^{2}$ of functions $v=\sum c_{\beta} \psi_{\beta} \in \tilde{L}_{\rho}^{2}$ with coefficients satisfying

$$
\sum\left|c_{\beta}\right|^{2}<\infty
$$

where the scalar product and the induced norm are given by

$$
(v, w)_{0}=\sum c_{\beta} a_{\beta} \text { for } w=\sum a_{\beta} \psi_{\beta} \in l_{\rho}^{2}, \quad \text { and }\|v\|_{0}^{2}=(v, v)_{0} .
$$

Obviously, $l_{\rho}^{2}$ is isomorphic to the Hilbert space $l^{2}$ of sequences $\left\{c_{\beta}\right\}$ with the same inner product, and hence

$$
\Phi \quad \text { is orthonormal in } l_{\rho}^{2} \text {. }
$$

It follows from (4.8) that

$$
l_{\rho}^{2} \subset \tilde{L}_{\rho}^{2} \quad \Longrightarrow \quad l_{\rho}^{2} \cap \tilde{L}_{\rho}^{2}=l_{\rho}^{2} .
$$

We next define a little Sobolev space $h_{\rho}^{2 k+1}$ of functions $v \in l_{\rho}^{2}$ such that $\mathbf{B} v \in l_{\rho}^{2}$, i.e.,

$$
\sum\left|\lambda_{\beta} c_{\beta}\right|^{2}<\infty \text {. }
$$

The scalar product and the induced norm in $h_{\rho}^{2 k+1}$ are

$$
(v, w)_{1}=(v, w)_{0}+(\mathbf{B} v, \mathbf{B} w)_{0} \quad \text { and } \quad\|v\|_{1}^{2}=(v, v)_{1} \equiv \sum\left(1+\left|\lambda_{\beta}\right|^{2}\right)\left|c_{\beta}\right|^{2} .
$$

This norm is equivalent to the graph norm induced by the positive operator $(-\mathbf{B}+a I)$ with $a>0$. Then $h_{\rho}^{2 k+1}$ is the domain of $\mathbf{B}$ in $l_{\rho}^{2}$. We also have a Sobolev embedding theorem,

$$
h_{\rho}^{2 k+1} \subset l_{\rho}^{2}{ }_{21} \text { compactly, }
$$


which follows from the well-known criterion of compactness in $l^{p}:$ a $T \subset l^{p}$ is compact iff

$$
\forall \varepsilon>0 \exists \text { integer } K=K(\varepsilon)>0 \text { such that } \forall\left\{c_{\beta}\right\} \in T \Longrightarrow \sum_{|\beta| \geq K}\left|c_{\beta}\right|^{p}<\varepsilon \text {; }
$$

see, e.g., [43].

Since the orthonormality of $\Phi$ is known to be of importance in operator theory and applications, in some linear and nonlinear problems dealing with operators like $\mathbf{B}$, the little space $l_{\rho}^{2}$ can play a special role in comparison with the big one $L_{\rho}^{2}$.

It follows from (4.11) that $\mathbf{B}$ is self-adjoint in $l_{\rho}^{2}$ with the domain $h_{\rho}^{2 k+1}$,

$$
(\mathbf{B} v, w)_{0}=(v, \mathbf{B} w)_{0} \quad \text { for all } v, w \in h_{\rho}^{2 k+1} .
$$

Notice that this a posteriori conclusion in a special functional setting is obtained after establishing all the necessary spectral properties of the operator.

\section{Spectrum And polynomial eigenfunctions of the ADJoint operator B*}

We now look to explicitly describe the eigenfunctions of the "adjoint" operator (3.25). We recall that $\mathbf{B}^{*}$ was derived in Section 3.5 via the blow-up scaling (3.24), and yet has nothing to do with the standard adjoint differential form $(\mathbf{B})^{*}$ in the metric of $L^{2}$, which, though is easily derived, has no further applications.

5.1. Indefinite metric. Thus, before we look at the operator $\mathbf{B}^{*}$ however, we first make the following easy observation:

Proposition 5.1. $\mathbf{B}^{*}$ is not adjoint to $\mathbf{B}$ in the standard metric of $L^{2}(\mathbb{R})$.

Proof. Let $v, w \in C_{0}^{\infty}(\mathbb{R})$, then integration by parts yields

$$
\begin{aligned}
\langle\mathbf{B} v, w\rangle & \equiv \int_{\mathbb{R}}\left((-1)^{k+1} v^{(2 k+1)}+\frac{1}{2 k+1}(y v)^{\prime}\right) w \mathrm{~d} y \\
& =\int_{\mathbb{R}}\left((-1)^{k} v^{(2 k)}-\frac{1}{2 k+1} y v\right) w^{\prime} \mathrm{d} y \\
& =\ldots=\int_{\mathbb{R}} v\left((-1)^{k} w^{(2 k+1)}-\frac{1}{2 k+1} y w^{\prime}\right) \mathrm{d} y \\
& =\left\langle v,(\mathbf{B})^{*} w\right\rangle, \quad \text { where } \quad(\mathbf{B})^{*}=(-1)^{k} D_{y}^{2 k+1}-\frac{1}{2 k+1} y D_{y} \neq \mathbf{B}^{*} .
\end{aligned}
$$

Thus, in order to get the correct adjoint operator $\mathbf{B}^{*}$, it is necessary to use another metric. The scalar product of this indefinite metric of the space $\bar{L}^{2}$ is given by

$$
\langle v, w\rangle_{*}=\int_{\mathbb{R}} v(y) \overline{w(-y)} \mathrm{d} y .
$$

Since $\mathbf{B}$ and $\mathbf{B}^{*}$ have real point spectrum (see (3.27), a full theory to be developed), we may omit the complex conjugate.

Proposition 5.2. $\mathbf{B}^{*}$ is adjoint to $\mathbf{B}$ in the indefinite metric of $\bar{L}^{2}(\mathbb{R})$, with the indefinite scalar product (5.1). 
Proof. For our operator, taking $v, w \in C_{0}^{\infty}(\mathbb{R})$

$$
\begin{aligned}
\langle\mathbf{B} v, w\rangle_{*} & =\int_{\mathbb{R}}\left((-1)^{k+1} v^{(2 k+1)}(y)+\frac{1}{2 k+1}(y v(y))^{\prime}\right) w(-y) \mathrm{d} y \\
& =\int_{\mathbb{R}}\left((-1)^{k+1} v^{(2 k)}(y)+\frac{1}{2 k+1} y v\right) w^{\prime}(-y) \mathrm{d} y \\
& =\ldots=\int_{\mathbb{R}} v(y)\left((-1)^{k+1} w^{(2 k+1)}(-y)-\frac{1}{2 k+1}(-y) w^{\prime}(-y)\right) \mathrm{d} y=\left\langle v, \mathbf{B}^{*} w\right\rangle_{*}
\end{aligned}
$$

Thus, $\mathbf{B}^{*}$ is adjoint to $\mathbf{B}$ in the given indefinite metric, which we write down again as

$$
\langle v, w\rangle_{*}=\int v(y) \overline{w(-y)} \mathrm{d} y \equiv\langle v, \overline{J w}\rangle, \quad \text { for any } v \in L_{\rho}^{2}, \quad J w \in L_{1 / \rho}^{2} .
$$

Here, the canonical symmetry operator $J w(y)=w(-y)$ is bounded, self-adjoint, and unitary (it is the Gramm operator of this metric). Moreover, one can see that, for the given anisotropic weight $\rho(y)$ in (3.17),

$$
J w \in L_{1 / \rho}^{2}, \quad \text { if } \quad w \in L_{\rho}^{2},
$$

so that the adjoint space in the indefinite metric $(\underline{5.2})$ is

$$
\left(L_{\rho}^{2}\right)_{*}^{*}=L_{\rho}^{2} .
$$

Let us next introduce another natural weighted space, with a symmetric exponentially decaying weight,

$$
L_{\rho^{*}}^{2}(\mathbb{R}), \quad \text { where } \quad \rho^{*}(y)=\frac{1}{\hat{\rho}(y)}=\mathrm{e}^{-a|y|^{\alpha}} \quad \text { in } \quad \mathbb{R} \text {, so }\left(L_{\hat{\rho}}^{2}\right)_{L^{2}}^{*}=L_{\rho^{*}}^{2} .
$$

Note that the standard "adjoint relation" of the weights $\rho^{*}(y)=\frac{1}{\rho(y)}$ exists for $y \ll-1$ only and is wrong for $y \gg 1$. Indeed, this reflects a strong anisotropic behaviour of the eigenfunctions $\psi_{\beta}(y)$ of $\mathbf{B}$ as $y \rightarrow \pm \infty$.

The set of even functions $E_{+}=\{v(-y) \equiv v(y)\}$ is a positive lineal (a linear manifold) of the metric (5.2),

$$
\langle v, v\rangle_{*}>0 \quad \text { for } \quad v \in E_{+} \subset L_{\hat{\rho}}^{2}, \quad v \neq 0,
$$

and odd functions $E_{-}=\{v(-y) \equiv-v(y)\} \subset L_{\hat{\rho}}^{2}$ give the corresponding negative lineal. Therefore, $L_{\hat{\rho}}^{2}$ with this metric is decomposable:

$$
v=v_{+}+v_{-} \equiv \frac{v(y)+v(-y)}{2}+\frac{v(y)-v(-y)}{2}, \quad \text { where } \quad v_{ \pm} \in E_{ \pm} \Longrightarrow L_{\hat{\rho}}^{2}=E_{+} \oplus E_{-},
$$

where, in addition, $E_{+} \perp E_{-}$in the metric (5.2). The corresponding positive majorizing metric is given by

$$
\left|\langle v, v\rangle_{*}\right| \leq[v, v]_{*} \equiv\left\langle v_{+}, v_{+}\right\rangle_{*}-\left\langle v_{-}, v_{-}\right\rangle_{*},
$$

etc. This case of the decomposable space with an indefinite metric having a straightforward majorizing one is treated as rather trivial; see Azizov-Iokhvidov [1] for linear operators theory in spaces with indefinite metric. Metric (5.2) is widely used therein; see [1, p. 13, 17, 23, 114]. Then the domain of $\mathbf{B}^{*}$ is defined as $H_{\rho^{*}}^{3}$, etc.

Historical Remark: As we mentioned, basic results of linear operator theory in spaces with indefinite metrics can be found in Azizov and Iokhvidov [1]. It was not until about 1944 that L.S. Pontryagin published the article on "Hermitian operators in spaces with 
indefinite metric" [47]. A new area of operator theory had been formed from Pontryagin's studies, which, during the time of the WWII, were originated and associated with some missile-type military research, [41]. This work set by Pontryagin was continued from 1949 and in the 1950s by M.G. Krein [39] and I.S. Iokhvidov [29].

5.2. Discrete spectrum and polynomial eigenfunctions of $\mathbf{B}^{*}$. We consider the spectrum of the linear adjoint operator $\mathbf{B}^{*}$ in the weighted space $L_{\rho^{*}}^{2}(\mathbb{R})$, with exponentially decaying weight (5.4). Here we have that $\rho^{*}(y)>0$ and $a \in\left(0,2 \hat{d}_{k}\right)$ (see (2.9)) is a sufficiently small constant. The proof of the following results does not differ from that of Lemma 4.1.

Lemma 5.1. $\mathbf{B}^{*}$ is a bounded linear operator from $H_{\rho^{*}}^{2 k+1}(\mathbb{R})$ to $L_{\rho^{*}}^{2}(\mathbb{R})$.

As we have mentioned before, we now, in an easier and direct manner, construct polynomial eigenfunctions of $\mathbf{B}^{*}$.

Lemma 5.2. (i) The operator (3.25) in $L_{\rho^{*}}^{2}$ has the same point spectrum $\sigma\left(\mathbf{B}^{*}\right)=\sigma(\mathbf{B})$ as in (4.6) and the eigenfunctions $\psi^{*}(y)$ are generalized Hermite polynomials given by

$$
\psi_{\beta}^{*}(y)=\frac{1}{\sqrt{\beta !}}\left[y^{\beta}+(-1)^{k+1} \sum_{j=1}^{\left\lfloor\frac{\beta}{2 k+1}\right\rfloor} \frac{1}{j !} D^{(2 k+1) j} y^{\beta}\right] .
$$

(ii) The eigenfunction subset $\Phi^{*}=\left\{\psi_{\beta}^{*}\right\}$ is complete in $L^{2}$ and in $L_{\rho^{*}}^{2}$.

Proof. (i) The construction is straightforward using the fact that the operator $y D_{y}$ is homogeneous. Consider the eigenfunction equation

$$
\mathbf{B}^{*} \psi_{\beta}^{*}=\lambda \psi_{\beta}^{*} \text {. }
$$

Assume that $\psi_{\beta}^{*}(y)$ is a polynomial of degree $l \geq 0$. Then we look for a solution of (5.6) in the form

$$
\psi_{\beta}^{*}(y)=\sum_{j=0}^{s} P_{j}(y)
$$

where each $P_{j}(y)$ is a homogeneous polynomial and $s=\left\lfloor\frac{l}{2 k+1}\right\rfloor$. From the eigenvalue problem (5.6), we can work out all terms of the polynomial, for a given degree $l$. Then

$$
(-1)^{k+1} D_{y}^{2 k+1} \psi_{\beta}^{*}-\frac{1}{2 k+1} y D_{y} \psi_{\beta}^{*}=\lambda_{\beta} \psi_{\beta}^{*} \quad \Longrightarrow \quad \lambda_{\beta}=-\frac{l}{2 k+1}, \quad l=0,1,2 \ldots,
$$

and we can define all other polynomials $P_{j}(y)$ by

$$
P_{j}(y)=\frac{(-1)^{(k+1)}}{j !} D^{(2 k+1) j} P_{0}(y), \quad j=1, \ldots, s .
$$

Fixing the leading term $P_{0}(y)=y^{\beta}$ calculating all the terms via (5.8) yields (5.5).

(ii) The proof of completeness is much simpler than that in Lemma 4.2(ii). In fact, it is a common fact in functional analysis that polynomials are complete in any reasonable weighted spaces; see the famous text-book [38, p. 431].

As in (4.4), we next define the space of $\Phi^{*}$-closure $\tilde{L}_{\rho^{*}}^{2}(\mathbb{R})$ with the corresponding property (4.5). Since, obviously, the resolvent now acts as follows:

$$
\text { for } v=\sum c_{\beta} \psi_{\beta}^{*}, \quad\left(\mathbf{B}^{*}-\lambda I\right)^{-1} v=\sum \frac{c_{\beta}}{\lambda_{\beta}-\lambda} \psi_{\beta}^{*},
$$


i.e., the convergence of the series is improved, it is not difficult to conclude that the resolvent is a compact operator in $\tilde{L}_{\rho^{*}}^{2}$, though we are not going to use such facts. By construction, (4.6) is the full spectrum of $\mathbf{B}^{*}$ in $\tilde{L}_{\rho^{*}}^{2}$, with the domain $\tilde{\mathcal{D}}\left(\mathbf{B}^{*}\right)=\tilde{L}_{\rho^{*}}^{2}(\mathbb{R}) \cap$ $H_{\rho^{*}}^{2 k+1}(\mathbb{R})$.

We need a few more properties. Firstly, we note that, as in [18, § 5.2], given an arbitrarily small $\varepsilon>0$, there holds

$$
c_{\beta}=o\left(|\beta|^{-|\beta|(\nu+\varepsilon)}\right) \Longrightarrow \sum c_{\beta} \psi_{\beta}^{*} \in \tilde{L}_{\rho^{*}}^{2} .
$$

In addition, if $v=\sum c_{\beta} \psi_{\beta}^{*} \in \tilde{L}_{\rho^{*}}^{2}$, then for arbitrarily small fixed $\varepsilon>0$,

$$
c_{\beta}=o\left(|\beta|^{-|\beta|(\nu-\varepsilon)}\right) \quad \text { for "almost all" }|\beta| \gg 1 \quad\left(\nu=\frac{2-\alpha}{2 \alpha}=\frac{2 k-1}{2(2 k+1)}\right) .
$$

In view of the fast decay (5.10) of the expansion coefficients, similar to $l_{\rho}^{2}$, we introduce the adjoint little Hilbert space $l_{\rho^{*}}^{2}$ of eigenfunction expansions $v=\sum c_{\beta} \psi_{\beta}^{*} \in \tilde{L}_{\rho^{*}}^{2}$ with the scalar product $(\cdot, \cdot)_{0 *}$ and the norm $\|\cdot\|_{0 *}$ defined as in (4.11). Then the domain of $\mathbf{B}^{*}$ in $l_{\rho^{*}}^{2}$ is the corresponding little Sobolev space $h_{\rho^{*}}^{2 k+1}$ compactly embedded into $l_{\rho^{*}}^{2}$. Here, $(\cdot, \cdot)_{1 *}$ and $\|\cdot\|_{1 *}$ denote the scalar product and the induced norm. Then $\mathbf{B}^{*}$ is self-adjoint in $l_{\rho^{*}}^{2}$, and $\tilde{L}_{\rho^{*}}^{2}, \tilde{H}_{\rho^{*}}^{2 k+1}$ are dense subspaces of $l_{\rho^{*}}^{2}$.

5.3. Bi-orthonormality of the bases $\Phi$ and $\Phi^{*}$. This is a principal issue for several applications of eigenfunction expansions via eigenfunction sets $\Phi$ and the adjoint one $\Phi^{*}$ to be developed later on. First of all, we note that the generalized Hermite polynomials $\psi_{\beta}^{*}(y) \in \Phi^{*}$ initially appeared in the semigroup expansions (3.10), (3.11) and (3.22), as more or less standard linear functionals defined in the weighted space $L_{\hat{\rho}}^{2}(\mathbb{R})$ as given in (3.13). Therefore, by Hilbert space theory, these polynomials belong to the true adjoint space: for any $\beta$,

$$
\psi_{\beta}^{*} \in L_{\hat{\rho}}^{2 *}(\mathbb{R})=L_{\rho^{*}}^{2}(\mathbb{R}), \quad \text { where } \quad \rho^{*}=\frac{1}{\hat{\rho}} \quad(\text { cf. (5.4) }) .
$$

Moreover, the whole set $\Phi^{*}$ of such polynomials is complete in $L_{\rho^{*}}^{2}$, and is closed in the corresponding space of $\Phi^{*}$-closure $\tilde{L}_{\rho^{*}}^{2}$.

However, this is not enough for further applications. Namely, let us return to the space of $\Phi$-closure $\tilde{L}_{\rho}^{2}(\mathbb{R})$ defined for B in Section 4.3. According to the construction therein, we have defined linear functionals from the adjoint space $\tilde{L}_{\rho}^{2 *}$. Thus, as we have seen earlier from the eigenfunction expansion of the semigroup (3.10) and (3.21) (for $\tau=0$ ), those linear functionals are well defined for data $u_{0} \in L_{\hat{\rho}}^{2}$ with the standard (not in the v.p. sense) definition of the integral; cf. (3.11). Therefore, we then need to extend those functionals to the whole space of $\Phi$-closure.

Before doing that, let us get rid of the indefinite metric $\langle\cdot, \cdot\rangle_{*}$, applying of which could rise some natural questions, and return to the standard (dual) $L^{2}$-one. Actually, we then need slightly revise our definition of eigenfunctions $\left\{\psi_{\beta}^{*}(y)\right\}$ of $\mathbf{B}^{*}$. Looking back at the expansion of the convolution, where the rescaled solution is given by (3.10), we note the 
definition of the moments of the initial data, which give rise to the adjoint eigenfunctions, $\left\{\psi_{\beta}^{*}(y)\right\}$. These moments (3.11), in the $L^{2}$-metric, are assumed to be given by

$$
M_{\beta}\left(u_{0}\right) \equiv\left\langle w_{0}(z), \psi_{\beta}^{*}(z)\right\rangle \equiv \frac{1}{\sqrt{\beta !}} \int_{\mathbb{R}} z^{\beta} u_{0}(z) \mathrm{d} z \quad \text { for any } \beta .
$$

However, we must have the representation in the indefinite metric that actually yields

$$
M_{\beta}\left(u_{0}\right) \equiv\left\langle w_{0}(z), \psi_{\beta}^{*}(z)\right\rangle_{*} \equiv \frac{1}{\sqrt{\beta !}} \int_{\mathbb{R}}(-z)^{\beta} u_{0}(z) \mathrm{d} z=\frac{(-1)^{\beta}}{\sqrt{\beta !}} \int_{\mathbb{R}} z^{\beta} u_{0}(z) \mathrm{d} z .
$$

Therefore, in the expansion there must be an extra multiplier $(-1)^{\beta}$ for the dual products. So, for purposes of convenience, instead of (5.5), we now have to take the adjoint eigenfunctions $\psi_{\beta}^{*}$ in the following form (i.e., the extra multiplier $(-1)^{|\beta|}$ is included):

$$
\hat{\psi}_{\beta}^{*}(y)=\frac{(-1)^{\beta}}{\sqrt{\beta !}}\left[y^{\beta}+(-1)^{(k+1)} \sum_{j=1}^{\left\lfloor\frac{\beta}{2 k+1}\right\rfloor} \frac{1}{j !} D^{(2 k+1) j} y^{\beta}\right] .
$$

Thus, as the next step, according to our construction, we first note that, by standard properties of Hilbert spaces, the space adjoint to $L_{\rho}^{2}(\mathbb{R})$ is

$$
L_{\rho}^{2 *}(\mathbb{R})=L_{\bar{\rho}}^{2}(\mathbb{R}), \quad \text { where } \quad \bar{\rho}=\frac{1}{\rho} \neq \rho^{*} \quad \Longrightarrow \quad \psi_{\beta}^{*} \notin L_{\rho}^{2 *}(\mathbb{R}) .
$$

Similarly, in the metric (5.2), for which (5.3) holds, there is no inclusion of polynomials either. In other words, unlike the more standard "parabolic" case [13, 18] (where always $\left.\rho^{*}=\frac{1}{\rho}\right)$, the polynomials $\psi_{\beta}^{*}$ do not belong to the adjoint space. However, we deal with the space of closure $\tilde{L}_{\rho}^{2} \subset L_{\rho}^{2}$, with the adjoint one $\tilde{L}_{\rho}^{2 *}$ being wider than the pure and standard $L_{\rho}^{2 *}$. Finally, completing this discussion of such intriguing aspects of functional spaces involved, which will not play a role later on, we mention that one needs to extend and define such "extended" linear functionals $\left\{\psi_{\beta}^{*}\right\}$ from the adjoint space $L_{\rho}^{2 *}$ to the space $L_{\rho^{*}}^{2}$, which are defined in the whole $\tilde{L}_{\rho}^{2}$. On one hand, this looks related to a standard procedure of extension of uniformly convex functional in linear normed spaces by the classic Hahn-Banach theorem1, [38].

On the other hand, naturally following our direct definition of the space of closure (4.4), which assumes the a priori knowledge of the expansion coefficients $\left\{c_{\beta}\right\}$ for any $v \in \tilde{L}_{\rho}^{2}$, such extended linear functionals for every $\beta$ act as follows:

$$
\left\langle v, \psi_{\beta}^{*}\right\rangle_{*} \equiv\left\langle v, \hat{\psi}_{\beta}^{*}\right\rangle \text { denote the expansion coefficient } c_{\beta} \text { of } v \text { in (4.5). }
$$

In view of the performed construction of $\tilde{L}_{\rho}^{2}$ via closure of finite sums, it is not difficult to see that such continuous extended linear functionals are defined uniquely (by the density of finite sums in (4.4)), and in $L_{\hat{\rho}}^{2}$ are given by the standard integrals as in (3.11) and (3.22). Defining a countable and complete set $\Phi^{*}$ of such extended linear functionals suffices for our applications.

\footnotetext{
1 "If $X$ is a linear normed space, $L$ is a linear manifold, and $f$ is a linear continuous functional defined on $L$, then $f$ can be extended to $F$ on $X$ and $\|F\|_{X}=\|f\|_{L} "$.
} 
Thus, overall, in the sense of (5.15), the standard bi-orthonormality of the bases $\left\{\psi_{\beta}\right\}$ and $\left\{\psi_{\beta}^{*}\right\}$ becomes trivial:

$$
\left\langle\psi_{\beta}, \psi_{\gamma}^{*}\right\rangle_{*} \equiv\left\langle\psi_{\beta}, \hat{\psi}_{\gamma}^{*}\right\rangle=\delta_{\beta \gamma} \quad \text { for any (multi-indices) } \beta \text { and } \gamma,
$$

where $\langle\cdot, \cdot\rangle$ is the usual duality product in $L^{2}(\mathbb{R})$ and $\delta_{\beta \gamma}$ is the Kronecker delta. Then, we treat similarly the adjoint linear functionals $\left\langle w, \psi_{\beta}\right\rangle_{*}$ for any $w \in \tilde{L}_{\rho^{*}}^{2}$, where $\Phi=\left\{\psi_{\beta}\right\}$ is now a countable (complete) set of linear functionals, which have been properly extended from the adjoint space $L_{\rho^{*}}^{2 *}(\mathbb{R})=L_{\bar{\rho}^{*}}^{2}(\mathbb{R})$, with $\bar{\rho}^{*}=\frac{1}{\rho^{*}}=\hat{\rho}$.

According to the above analysis, we now show some convenient formal calculus with non-convergent integrals, which illustrate standard bi-orthonormality and other properties. For instance, according to these formal integration techniques, we have:

\section{Proposition 5.3.}

$$
\left\langle\psi_{\beta}, \psi_{\beta}^{*}\right\rangle_{*}=1 \text { for all } \beta \geq 0 .
$$

Proof. We start by looking at the scalar product, in the indefinite metric, defined by

$$
\left\langle\psi_{\beta}, \psi_{\beta}^{*}\right\rangle_{*}=-\int \psi_{\beta}(y) \psi_{\beta}^{*}(-y) \mathrm{d} y .
$$

By our definitions of $\psi_{\beta}(y)\left(\right.$ (5.13) ) and $\psi_{\beta}^{*}(y)$ (5.5), we substitute to find that

$$
\left\langle\psi_{\beta}, \psi_{\beta}^{*}\right\rangle_{*}=-\int \frac{1}{\sqrt{\beta !}} D_{y}^{\beta} F(y) \frac{1}{\sqrt{\beta !}}\left[(-y)^{\beta}+(-1)^{(k+1)} \sum_{j=1}^{\left\lfloor\frac{\beta}{2 k+1}\right\rfloor} \frac{1}{j !} D^{(2 k+1) j}(-y)^{\beta}\right] \mathrm{d} y .
$$

We now apply to $\psi_{\beta}^{*}$ the identity operator $\left(D_{y}^{\beta}\right)^{-1} D_{y}^{\beta}=I$, with a standard definition and construction of the inverse integral operator $\left(D_{y}^{\beta}\right)^{-1}=D_{y}^{-\beta}$, such that

$$
\left\langle\psi_{\beta}, \psi_{\beta}^{*}\right\rangle_{*}=\left\langle\psi_{\beta},\left(D_{y}^{-\beta} D_{y}^{\beta}\right) \psi_{\beta}^{*}\right\rangle_{*} .
$$

Integrating by parts, $\beta$ times, we find that

$$
\begin{aligned}
\left\langle\psi_{\beta}, \psi_{\beta}^{*}\right\rangle_{*} & =\int \frac{1}{\sqrt{\beta !}} D_{y}^{\beta} F(y)\left(D_{y}^{-\beta} D_{y}^{\beta}\right) \frac{1}{\sqrt{\beta !}}\left((-y)^{\beta}+o\left(y^{\beta}\right)\right) \mathrm{d} y \\
& =\frac{1}{\beta !} \int(-1)\left[D_{y}^{\beta-1} F(y) D_{y}^{-\beta+1} D_{y}^{\beta}\left((-y)^{\beta}+o\left(y^{\beta}\right)\right)\right] \mathrm{d} y \\
& =\ldots=\frac{1}{\beta !} \int(-1)^{\beta}\left[F(y) D_{y}^{\beta}\left((-y)^{\beta}+o\left(y^{\beta}\right)\right)\right] \mathrm{d} y \\
& =\frac{1}{\beta !} \int(-1)^{\beta} F(y)(-1)^{\beta} \beta ! \mathrm{d} y .
\end{aligned}
$$

One can see that, according to these formal calculus of integration by parts, we each time improve the convergence properties of the integrals involved, meaning using a distributional treatment of those integrals as values of certain linear functionals as generalized functions (distributions). As customary, this corresponds to a regularization of divergent integrals.

Hence, eventually, it follows that

$$
\left\langle\psi_{\beta}, \psi_{\beta}^{*}\right\rangle_{*}=\int F(y) \mathrm{d} y=1,
$$

so finally we arrive at a convergent integral, but not absolutely, since $F$ is not Lebesgue measurable in $\mathbb{R}$, as we have seen. 
Proposition 5.4. In terms of the above formal calculus,

$$
\left\langle\psi_{\beta}, \psi_{\gamma}^{*}\right\rangle_{*}=0 \text { for all } \beta \neq \gamma \text {. }
$$

Proof. The first part of the proof follows that of Proposition 5.3. First, consider the case where $\beta>\gamma$. After integration by parts, it can be seen that $\left\langle\psi_{\beta}, \psi_{\gamma}^{*}\right\rangle_{*}$ may be written as

$$
\left\langle\psi_{\beta}, \psi_{\gamma}^{*}\right\rangle_{*}=(-1)^{\beta} \int \frac{(-1)^{\beta}}{\sqrt{\beta !}}\left(D_{y}^{\beta}\right)^{-1} D_{y}^{\beta} F(y) \frac{1}{\sqrt{\gamma !}} D_{y}^{\beta}\left((-y)^{\gamma}+\ldots\right) \mathrm{d} y .
$$

However, since $\beta>\gamma$, then it is known that $D_{y}^{\beta}(-y)^{\gamma}=0$. So it follows that (5.17) holds.

Now consider the case when $\beta<\gamma$. In this case it can easily be seen that the above argument will not work. Rather than attempting to use a similar argument, we instead use another proof which encompasses both cases of $\beta>\gamma$ and $\beta<\gamma$.

By the definitions of the linear operator $\mathbf{B}$ and the adjoint operator $\mathbf{B}^{*}$, we know that

$$
\left\{\begin{array}{l}
\mathbf{B} \psi_{\beta}=\lambda_{\beta} \psi_{\beta}, \\
\mathbf{B}^{*} \psi_{\gamma}^{*}=\lambda_{\gamma} \psi_{\gamma}^{*},
\end{array}\right.
$$

which defines the eigenvalue problems for these two operators. Taking the inner product of (5.18), in the indefinite metric, with $\psi_{\gamma}^{*}$ and $\psi_{\beta}$, respectively, yields

$$
\left\{\begin{array}{l}
\left\langle\mathbf{B} \psi_{\beta}, \psi_{\gamma}^{*}\right\rangle_{*}=\lambda_{\beta}\left\langle\psi_{\beta}, \psi_{\gamma}^{*}\right\rangle_{*} \\
\left\langle\psi_{\beta}, \mathbf{B}^{*} \psi_{\gamma}^{*}\right\rangle_{*}=\lambda_{\gamma}\left\langle\psi_{\beta}, \psi_{\gamma}^{*}\right\rangle_{*} .
\end{array}\right.
$$

However from the definition of the adjoint operator $\mathbf{B}^{*}$, we know that

$$
\left\langle\mathbf{B} \psi_{\beta}, \psi_{\gamma}^{*}\right\rangle_{*}=\left\langle\psi_{\beta}, \mathbf{B}^{*} \psi_{\gamma}^{*}\right\rangle_{*} .
$$

Hence, if $\beta \neq \gamma$ (i.e., $\lambda_{\beta} \neq \lambda_{\gamma}$ ), (5.17) follows.

\section{Semilinear Dispersion PDEs: VSSs, Numerics, AND PRELIMINARIES}

6.1. Semilinear model. We now consider the odd-order problem, but now with a nonlinear absorption. We look at the Cauchy problem for the semilinear odd-order equation (1.20), for $k=1,2,3, \ldots$, with sufficiently good initial data $u(x, 0)=u_{0}(x)$. As we have already commented on, this non-integrable model is somehow connected to the KdV equation (1.4), with the difference being that the extra operator $-|u|^{p-1} u$, which corresponds to absorption, is simpler and of zero differential order. This kind of nonlinearity also allows us to avoid entering remarkable classes of integrable PDEs, which obey various extremely strong and quite specific properties that are illusive for more general nonlinear odd-order PDEs.

Let us mention again, as in the linear case, even-order semilinear problems have been studied over recent years and are fairly well understood, unlike similar odd-order ones. The related generalized semilinear even-order model is given by

$$
u_{t}=-(-\Delta)^{m} u \pm|u|^{p-1} u,
$$

which includes both the cases of absorption and reaction. The lower order absorption case, with $m=1$, corresponds to the heat equation with absorption given in (1.13). 
6.2. Similarity solutions of semilinear equations. As is customary in PDE theory, there exists a critical exponent for (1.20) given by

$$
p=p_{0}=1+\left.\frac{2 k+1}{N}\right|_{N=1}=2 k+2 .
$$

It can be called the critical Fujita exponent; see further comments below. As for the parabolic equation (6.1), where

$$
p_{0}=1+\frac{2 m}{N}
$$

(as in (6.2), $2 m$ stands for the order of the differential operator involved), the critical Fujita exponent characterizes parameter ranges of blow-up and non-blow-up solutions and changing of the stability of the trivial zero solutions for the PDEs under consideration.

As usual, we consider self-similar solutions of the very singular type of (1.20)

$$
u_{*}(x, t)=t^{-\frac{1}{p-1}} f(y), \quad y=x / t^{\frac{1}{2 k+1}},
$$

where $f$ solves the ODE

$$
(-1)^{k+1} f^{(2 k+1)}+\frac{1}{2 k+1} f^{\prime} y+\frac{1}{p-1} f-|f|^{p-1} f=0 \quad \text { in } \quad \mathbb{R} .
$$

Unlike the linear case, we cannot integrate to reduce the order of the ODE and hence we remain with an equation of order $2 k+1$. We see that (6.3) is a difficult higher-order equation and so begin with numerical results.

6.3. Numerical results for the semilinear equation: $k=1$. We look at similarity profiles of the semilinear equation (1.6), for $k=1$,

$$
f^{\prime \prime \prime}+\frac{1}{3} f^{\prime} y+\frac{1}{p-1} f-|f|^{p-1} f=0 \quad \text { in } \quad \mathbb{R} .
$$

Once again we use the MatLab bvp4c solver to plot the profiles. This however gives us the same problem, as in Section [2.3, of solving an initial value problem using the BVP solver. We take $f$ and $f^{\prime}$ to be zero, as boundary conditions at the left-hand boundary point of a fixed interval and $f$ to be zero as the condition at the right-hand point. This corresponds well to a proper BVP setting for odd-order operators; see [14] as a guide. We then try to shift the point at the right boundary in order to coincide with a point where the oscillations go through zero and hence find the best profile. We look in particular for convergence and reflectional (about the axis $f=0$ ) symmetry of the tail.

Figures 4 and 5 show two sets of profiles for $p=1.9$ and $p=3.3$. Whilst we cannot guarantee the accuracy of the size of the tail, the profile close to the origin, where max $|f|$ is obtained, is very stable. We see that as $p$ decreases, $\max |f|$ increases and this seems to justify the term "Very Singular", with the mass concentrated close to $y=0$.

In the linear case set out in Section 2.3, due to the instability of the solutions, we had to use a "matching technique" in order to find reliable profiles. However, in the semilinear case, this is not necessary, as the profiles are stable, since we do not have a scaling group of solutions $\{c f\}$, due to the nonlinear term $|f|^{p-1} f$. In fact, we find that whilst the tail of the profile may differ, given different boundary points, the rest of the structure is extremely stable and rarely changes. For, approximately,

$$
2 \leq \underset{29}{p} \leq 3
$$


we can find profiles easily, for almost every boundary point value we use and they only really differ from the tail. But for other values it is more difficult to find profiles, especially reliable ones.

In particular, we look to see the behaviour of the solutions as $p \rightarrow 1^{+}$and as $p \rightarrow 4^{-}$, which is the critical Fujita exponent (6.2) for $k=1$.

Since we are using the boundary value solver of MatLab, we face numerous difficulties in obtaining the correct numerical results. As we are solving the initial value problem of the semilinear equation as a boundary value problem, difficulties arise in finding the correct right-hand end-point of the interval.

As we do not know exactly at which points $f$ and $f^{\prime}$ are both zero, we attempt to approximate the point by looking at various profiles and finding the best. If the far right boundary point is incorrect, then we produce artificial oscillations. We know which profiles are most likely to be false from the analysis we have done on odd-order linear PDEs. Since the semilinear equation can be naturally treated as the linear one with a perturbation $-|f|^{p-1} f$, we expect similar behaviour. With a wrong boundary point, we can end up with non-symmetric tails, since we are forcing the oscillations through a specific point. Hence, the most important condition we look for in reliable profiles are that the oscillations are symmetric.

We also look to ensure that the oscillations become symmetric as quickly as possible. This also follows from having forced oscillations due to wrong boundary points. Finally, we look for oscillations that decay as $y$ increases.

The solver that we use, bvpinit, requires as the name suggests an initial guess for the function. Whilst this guess does not necessarily have to be very accurate, occasionally a wrong guess can lead to wrong profiles being found. We have yet to find any evidence so far, to suggest that the initial guess is bad enough to affect the profiles though. However for less stable profiles where $p \sim 2$, the solutions blow-up and the initial guess for $\max |f|$ may not be accurate. The values needed for the initial guess for the maximum value are much larger than the maximum value found in the profile. Whilst the profiles look reliable, it is unclear as to whether the inaccurate initial guess affects the output.

Thus, the conditions we look at to ensure the best possible profiles can be briefly summarised as follows:

- Symmetry (reflectional, $f \mapsto-f$ ) of tail for $y \gg 1$.

- Symmetry of tail occurs as close to 0 as possible.

- Minimisation of symmetric tail.

Finally, in Figure 6 we present the VSS profile for $p=3.99$, which is sufficiently close to the bifurcation critical Fujita exponent $p_{0}=4$; see Section 7.4 for derivation.

6.4. Numerical results: $k \geq 2$. For $k=2$, scaling (2.1) in the PDE yields the following equation for $f$ :

$$
u_{t}=-u_{x x x x x}-|u|^{p-1} u \quad \Longrightarrow \quad-f^{(5)}+\frac{1}{5} f^{\prime} y+\frac{1}{p-1} f-|f|^{p-1} f=0 \quad \text { in } \quad \mathbb{R} .
$$




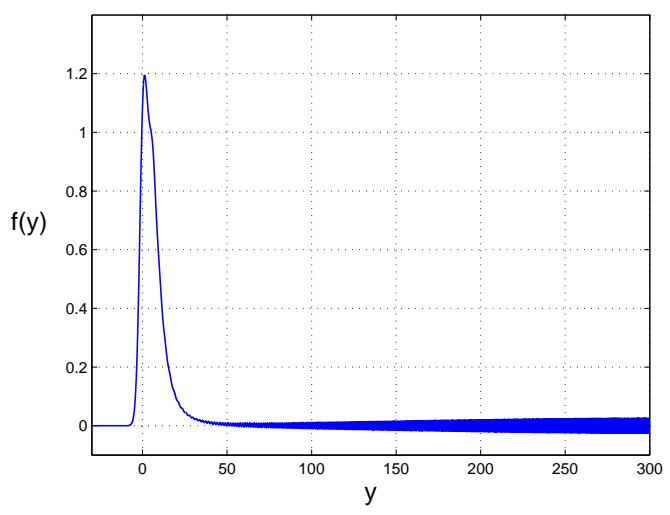

(a) General view of $f(y)$.

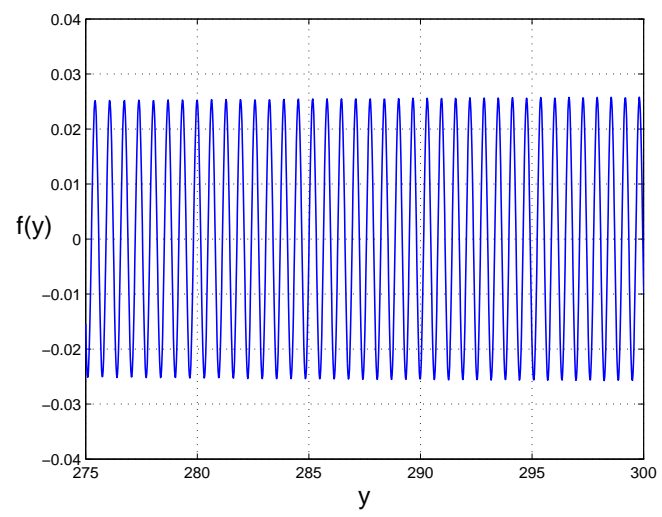

(b) "Tail" of the solution, for $y \gg 1$

Figure 4. A VSS profile of (6.4) for $p=1.9$.

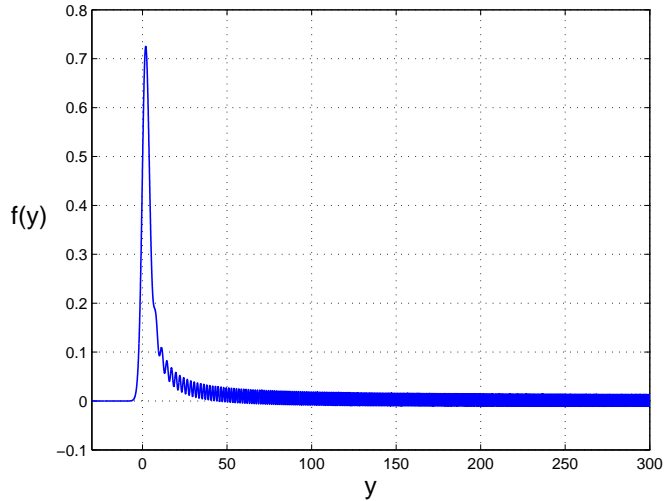

(a) General view of $f(y)$

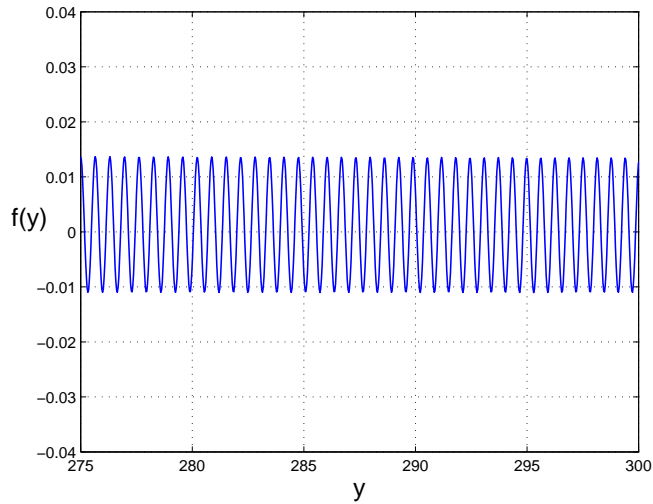

(b) "Tail" of the solution, for $y \gg 1$

Figure 5. A VSS profile of (6.4) for $p=3.3$.

In Figure 7, we show the similarity solution of the ODE (6.5) for $p=4$, where the tail is not that oscillatory as used to be for $k=1$. Figure 8 shows VSS profiles for $p=3$ and $p=5.9$, which is slightly lower than the critical exponent $p_{0}=6$ for $k=2$.

Finally, we present a few VSS profiles for the seventh-order equation for $k=3$ :

$$
u_{t}=u_{x x x x x x x}-|u|^{p-1} u \quad \Longrightarrow \quad f^{(7)}+\frac{1}{7} f^{\prime} y+\frac{1}{p-1} f-|f|^{p-1} f=0 \quad \text { in } \quad \mathbb{R} .
$$

These are given in Figure 9 for $p=4,5$, and 6 . Note that the first critical exponent is $p_{0}=8$ for $k=3$, so these values are well below the $p_{0}$-bifurcation point; see Section 7.4 . 


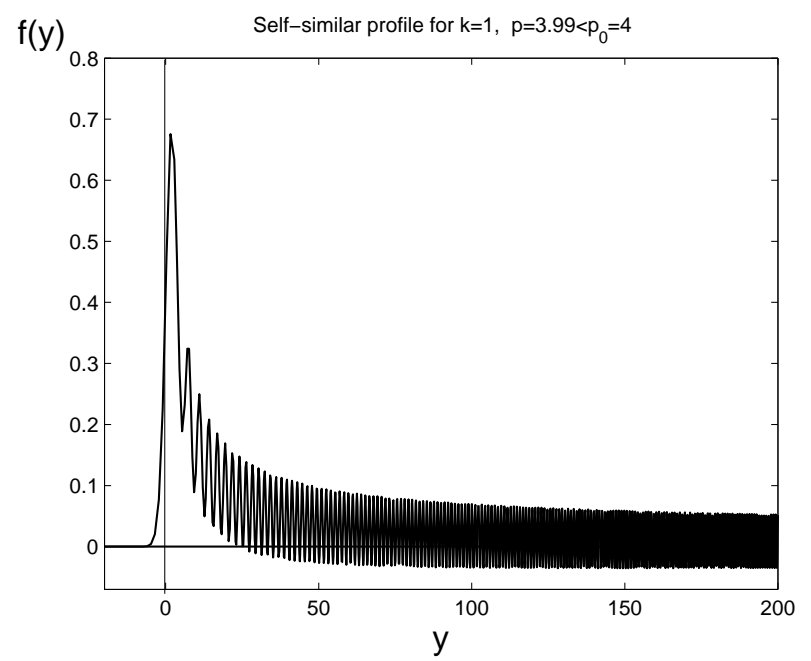

Figure 6. A VSS profile $f(y)$ of (6.4) for $p=3.99<p_{0}=4$.

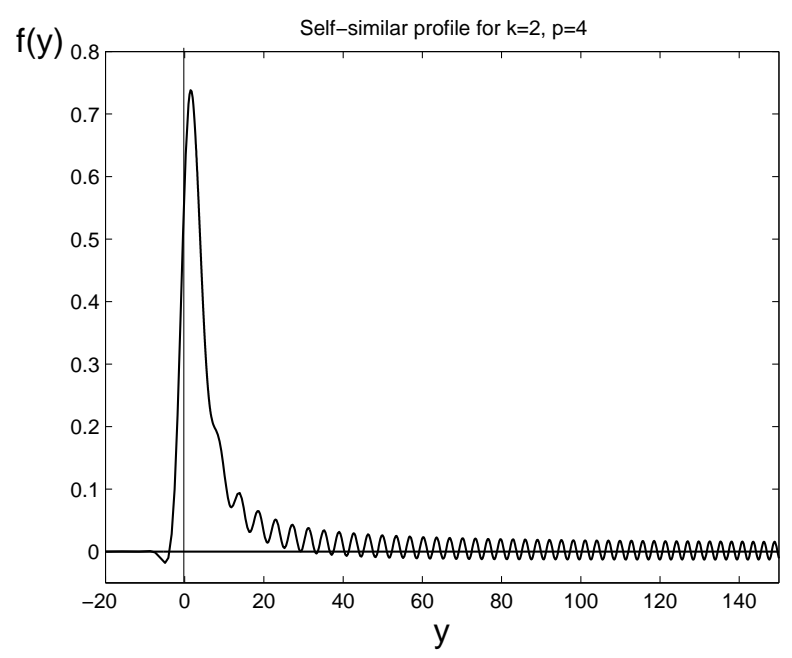

Figure 7. A VSS profile $f(y)$ of (6.5) for $p=4<p_{0}=6$.

\section{LINEARIZED STABILITY ANALYSIS AND CENTRE SUBSPACE BEHAVIOUR: APPLICATIONS OF HERMITIAN SPECTRAL THEORY}

7.1. Linearized stability and critical (Fujita) exponent. We begin with some applications of the spectral analysis obtained before, in the theory for LDEs.

As in the linear case, we introduce the following similarity scaling in the semilinear equation (1.20), with

$$
u(x, t)=(1+t)^{-\frac{1}{2 k+1}} v(y, \tau), \quad y=x /(1+t)^{\frac{1}{2 k+1}}, \quad \tau=\ln (1+t) .
$$

Our rescaled equation is then given by

$$
v_{\tau}=(-1)^{k+1} D_{y}^{2 k+1} v+\frac{1}{2 k+1} y D_{y} v+\frac{1}{p-1} v-|v|^{p-1} v \equiv \mathbf{B}_{1} v-|v|^{p-1} v .
$$




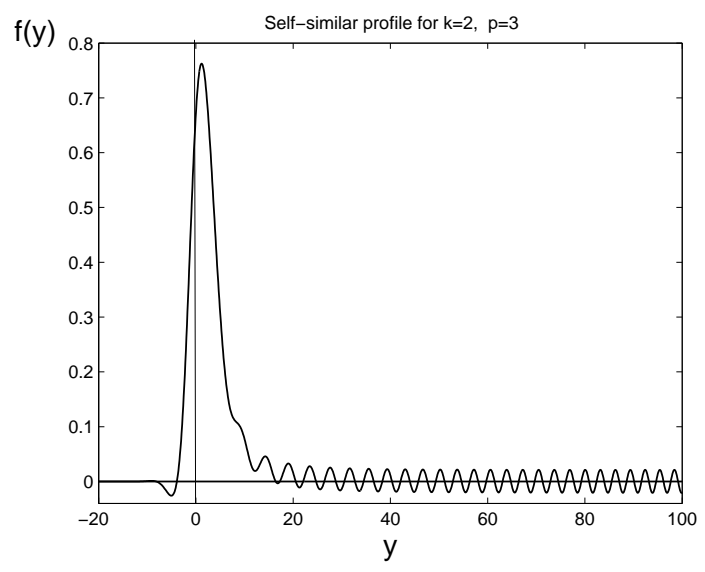

(a) $p=3$

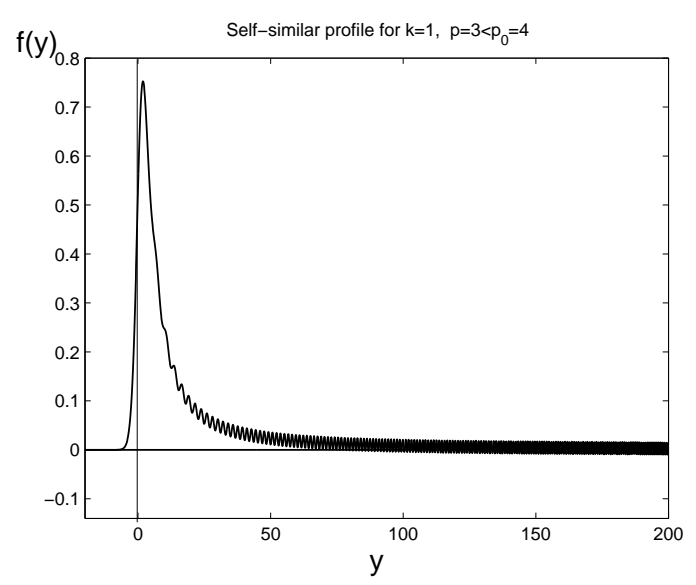

(b) $p=5.9$

Figure 8. VSS profiles $f(y)$ of (6.5) for $p=3$ (a) and $p=5.9<p_{0}=6$ (b).

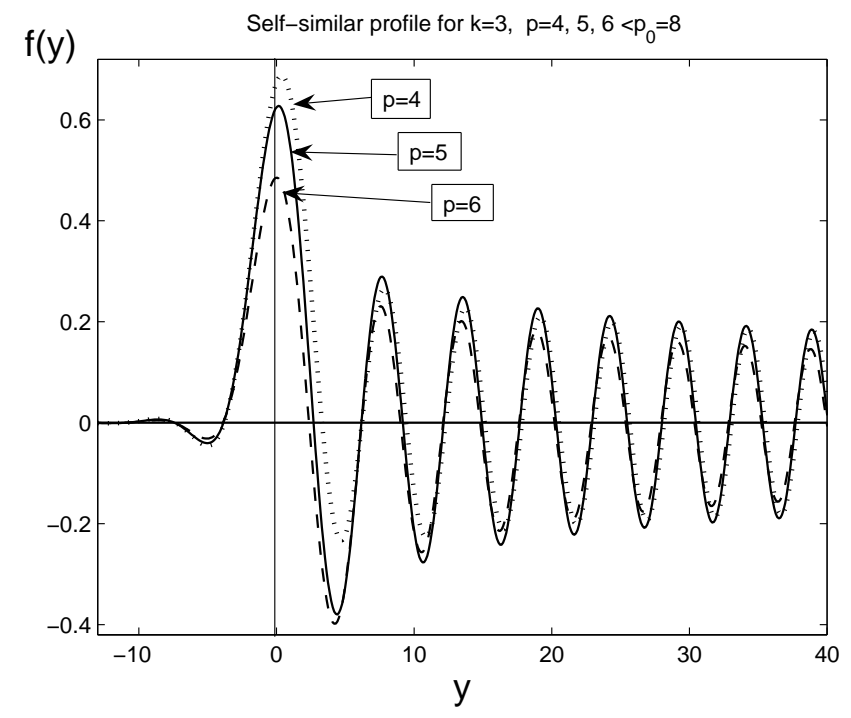

Figure 9. VSS profiles of the ODE (6.6) for $p=4,5,6$.

Here, the linear operator $\mathbf{B}_{1}$ is defined by

$$
\mathbf{B}_{1}=\mathbf{B}+d_{1} I, \quad \text { where } \quad d_{1}=\frac{1}{p-1}-\frac{1}{2 k+1}=\frac{p_{0}-p}{(2 k+1)(p-1)},
$$

and $\mathbf{B}$ is our canonical rescaled one (2.2).

Lemma 7.1. For $p>p_{0}=2 k+2$, zero is exponentially linearly stable for (7.1) in $H_{\rho}^{2 k+1}(\mathbb{R}) \cap \tilde{L}_{\rho}^{2}(\mathbb{R})$.

Proof. We look at the linearized problem of (17.1) about zero,

$$
v_{\tau}=\mathbf{B}_{13} v
$$


Then the spectrum is given by $\sigma\left(\mathbf{B}_{1}\right)=\left\{d_{1}-\frac{l}{2 k+1}\right.$ for $\left.l \geq 0\right\}$. Therefore, for $l=0$, $\lambda_{0}=d_{1} \equiv \frac{1}{p-1}-\frac{1}{2 k+1}<0$, for all $p>2 k+2$. So, in our space,

$$
\|v(\tau)\|_{2 k+1, \rho} \sim \mathrm{e}^{\lambda_{0} \tau} \rightarrow 0 \quad \text { as } \quad \tau \rightarrow \infty .
$$

Hence zero is linearly stable for $p>p_{0}$.

Lemma 7.2. For $1<p<p_{0}$, zero is exponentially linearly unstable for (17.1), for small data $v_{0} \in H_{\rho}^{2 k+1} \cap \tilde{L}_{\rho}^{2}(\mathbb{R})$.

Proof. For $l=0, \lambda_{0}=d_{1} \equiv \frac{1}{p-1}-\frac{1}{2 k+1}>0$, so that

$$
\|v(\tau)\|_{2 k+1, \rho} \sim \mathrm{e}^{\lambda_{0} \tau} \rightarrow+\infty
$$

Hence zero is linearly unstable for $p<p_{0}$.

Thus, $p_{0}=2 k+2$ plays almost a full role of the critical Fujita exponent for (1.20), that mimics the standard one $p_{0}=1+\frac{2 m}{N}(=2 m+1$ for $N=1)$ for the semilinear parabolic equations (6.1); see [13] for extra details.

7.2. Centre subspace behaviour. Let us show that, at some critical values of $p$, there exists some quite special asymptotics of solutions.

FIRST CRITICAL EXPONENT $p_{0}$. We look again at the rescaled equation given by (17.1).

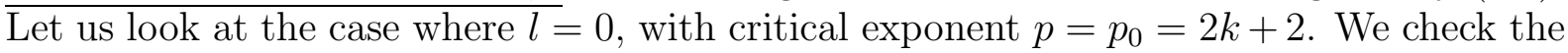
behaviour close to the centre subspace of $\mathbf{B}$, i.e.,

$$
v(\tau)=c_{0}(\tau) \psi_{0}+v_{0}^{\perp}(\tau)
$$

where $v_{0}^{\perp}$ is asymptotically small in comparison with the first term and is orthogonal to $\psi_{0}$, i.e., $\left\langle v_{0}^{\perp}, \psi_{k}^{*}\right\rangle_{*}=0$, for any $k \geq 1$. Then, since $\mathbf{B} \psi_{0}=0$, we multiply the equation by $\psi_{0}^{*} \equiv 1$ to get the following leading term:

$$
c_{0}^{\prime}=-\left|c_{0}\right|^{2 k+1} c_{0}\left\langle\left|\psi_{0}\right|^{2 k+1} \psi_{0}+\ldots, \psi_{0}^{*}\right\rangle_{*}
$$

We need to assume that (see a justification below)

$$
\gamma_{0}=\left\langle\left|\psi_{0}\right|^{2 k+1} \psi_{0}, \psi_{0}^{*}\right\rangle_{*}>0
$$

Note that analytically, proving that $\gamma_{0} \neq 0$ is difficult and even checking this numerically is also questionable. So, assuming that $\gamma_{0}>0$,

$$
\left|c_{0}\right|^{-(2 k+1)} \frac{c_{0}^{\prime}}{c_{0}}=-\gamma_{0}+\ldots \quad \Longrightarrow \quad-\frac{1}{2 k+1}\left|c_{0}\right|^{-(2 k+1)}=-\gamma_{0} \tau+\ldots \quad \text { as } \quad \tau \rightarrow \infty .
$$

Finally, this yields the following rate of decay:

$$
c_{0}(\tau) \approx\left[(2 k+1) \gamma_{0} \tau\right]^{-\frac{1}{2 k+1}} \text { for } \tau \gg 1
$$

Thus, the centre subspace behaviour of $u(x, t)$ is given, as $t \rightarrow \infty$, by

$$
u(x, t) \approx(1+t)^{-\frac{1}{2 k+1}}\left[(2 k+1) \gamma_{0} \ln (1+t)\right]^{-\frac{1}{2 k+1}} \psi_{0}\left(x /(1+t)^{\frac{1}{2 k+1}}\right),
$$

i.e., contains a typical extra logarithmic factor. A full justification of such a behaviour remains open (even existence of an invariant centre manifold is obscure). 
OTHER CRITICAL EXPONENTS $p_{l}$. Let us now look at the general case for $l$, with critical point $p=p_{l}=1+\frac{2 k+1}{l+1}$, where we check the behaviour close to the one-dimensional kernel of $\mathbf{B}-\lambda_{l} I$, by setting

$$
v=c_{l}(\tau) \psi_{l}+v_{l}^{\perp}
$$

Here, $v_{l}^{\perp}$ is small and orthogonal to $\psi_{l}$, as before. Then for $\mathbf{B} \psi_{l}=\lambda_{l} \psi_{l}$, we take the inner product with $\psi_{l}^{*}$ to get, for $\tau \gg 1$,

$$
c_{l}^{\prime} \approx-\left|c_{l}\right|^{\frac{2 k+1}{l+1}} c_{l}\left\langle\left|\psi_{l}\right|^{\frac{2 k+1}{l+1}} \psi_{l}, \psi_{l}^{*}\right\rangle_{*} .
$$

For convenience, let us assume, as usual, that

$$
\gamma_{l}=\left\langle\left|\psi_{l}\right|^{\frac{2 k+l}{l+1}} \psi_{l}, \psi_{l}^{*}\right\rangle_{*}>0
$$

Hence, assuming also that $c_{l}(\tau)>0$, we have, for $\tau \gg 1$,

$$
c_{l}^{-\frac{2 k+2+l}{l+1}} c_{l}^{\prime} \approx-\gamma_{l} \quad \Longrightarrow \quad-\frac{l+1}{2 k+1} c_{l}^{-\frac{2 k+1}{l+1}} \approx-\gamma_{l} \tau \quad \Longrightarrow \quad c_{l}(\tau) \approx\left(\frac{2 k+1}{l+1} \gamma_{l} \tau\right)^{-\frac{l+1}{2 k+1}}
$$

Hence, the stable subspace behaviour of $u(x, t)$ as $t \rightarrow \infty$, for all critical exponents $p=p_{l}$, is given by

$$
u(x, t) \approx(1+t)^{-\frac{1+l}{2 k+1}}\left[\frac{2 k+1}{l+1} \gamma_{l} \ln (1+t)\right]^{-\frac{l+1}{2 k+1}} \psi_{l}\left(x /(1+t)^{\frac{1}{2 k+1}}\right) .
$$

So, there exists a countable set of stable subspace behaviours governed by eigenfunctions, corresponding to the point spectrum $\sigma(\mathbf{B})=\left\{-\frac{l}{2 k+1}, \quad l \geq 0\right\}$.

7.3. Why $\gamma_{l}>0$ : numerics. For the above analysis to hold, it is assumed that

$$
\gamma_{l}=\left\langle\left|\psi_{l}\right|^{\frac{2 k+1+l}{l+1}} \psi_{l}, \psi_{l}^{*}\right\rangle_{*}>0
$$

which is not an easy inequality to prove. Note that, for all $l \geq 0$, the eigenfunctions $\psi_{l}(y)$ and $\psi_{l}^{*}(y)$ are of the same parity, so that the indefinite metric $\langle\cdot, \cdot\rangle_{*}$ plays no role here and can be replaced by the standard $L^{2}$-one (for odd l's, with the minus sign).

Let us first look at the case $l=0$. We need to show that

$$
\gamma_{0}=\left\langle\left|\psi_{0}\right|^{2 k+1} \psi_{0}, \psi_{0}^{*}\right\rangle_{*}>0 .
$$

However, in this case we have that $\psi_{0}^{*} \equiv 1$, so in essence it is enough to prove that

$$
\gamma_{0}=\int\left|\psi_{0}\right|^{2 k+1} \psi_{0}>0
$$

Attempting to prove this rigorously, is very difficult as well. However, numerically it can be shown, for $k=1$ at least, that this inequality is true.

Certainly, we know that the first eigenfunction $\psi_{0} \equiv F(y)$ can be found, for the lowerorder case $k=1$. Using the MatLab function trapz, which uses a trapezoidal method of integration, the integral can be approximated to

$$
\int|F|^{3} F=0.0300 \ldots>0 \text {. }
$$

It is noted that, since this numerical solution $F$, is not the fundamental kernel satisfying $\int F=1$, we scale the calculations such that this is true. So (7.3) holds for the true rescaled fundamental kernel. 
Solving the problem (7.2) for $k \geq 2$ is more difficult as the shooting problem to find further eigenfunctions is not easy, but not impossible. Similarly, it is possible to construct solutions for all $\psi_{l}$, where $l \geq 0$. Again the problem of shooting is difficult, but not impossible to justify the inequality (7.2).

7.4. Bifurcation points and $p$-diagrams. Once again, we look at our semilinear equation given in (6.3), which we can write now as

$$
\mathbf{B} f+\left(\frac{1}{p-1}-\frac{1}{2 k+1}\right) f-|f|^{p-1} f=0 \Longleftrightarrow \mathbf{B}_{1} f-|f|^{p-1} f=0,
$$

where $\mathbf{B}_{1}=\mathbf{B}+d_{1} I$ is defined as before. Critical exponents $\left\{p_{l}\right\}$ occur when

$$
d_{1} \equiv \frac{1}{p_{l}-1}-\frac{1}{2 k+1}=-\lambda_{l}, \quad \text { i.e., when } \quad \frac{1}{p_{l}-1}-\frac{1}{2 k+1}=\frac{l}{2 k+1} \text {. }
$$

Therefore our critical exponents, $p_{l}$, are given by

$$
p_{l}=1+\frac{2 k+1}{l+1}, \quad l=0,1,2, \ldots .
$$

We can see from this that $p_{l} \rightarrow 1^{+}$as $l \rightarrow+\infty$.

We look at $p$ near these critical values, so that $p \approx p_{l}$. We set $\varepsilon=p_{l}-p$, and then

$$
\left(\mathbf{B}-\lambda_{l} I\right) f+\varepsilon a_{0} f=|f|^{p-1} f+O\left(\varepsilon^{2}\right), \quad \text { where } \quad a_{0}=\frac{1}{\left(p_{l}-1\right)^{2}}=\left(\frac{l+1}{2 k+1}\right)^{2} .
$$

By classic bifurcation theory [56], our solution $f$ can be given by

$$
f=C \psi_{l}+w^{\perp}
$$

where $w^{\perp}$ is orthogonal to $\psi_{l}$,

$$
\left\langle w^{\perp}, \psi_{l}^{*}\right\rangle_{*}=0 .
$$

Thus, taking the inner product of (17.4) with $\psi_{l}^{*}$, we have that

$$
a_{0} \varepsilon C\left\langle\psi_{l}, \psi_{l}^{*}\right\rangle_{*}=|C|^{p-1} C\left\langle\left|\psi_{l}\right|^{p-1} \psi_{l}, \psi_{l}^{*}\right\rangle_{*} .
$$

Since $\left\langle\psi_{l}, \psi_{l}^{*}\right\rangle_{*}=1$, we find

$$
|C|^{p-1}=\frac{a_{0} \varepsilon}{\left\langle\left|\psi_{l}\right|^{p-1} \psi_{l}, \psi_{l}^{*}\right\rangle_{*}}=\frac{1}{\kappa_{l}}\left(\frac{l+1}{2 k+1}\right)^{2} \varepsilon, \quad \text { where } \quad \kappa_{l}=\left\langle\left|\psi_{l}\right|^{p-1} \psi_{l}, \psi_{l}^{*}\right\rangle_{*} .
$$

For $p=1$, we have that $\kappa_{l}=\left\langle\psi_{l}, \psi_{l}^{*}\right\rangle_{*}=1$, and so by continuity with respect to $p$, we must have that

$$
\kappa_{l}>0 \text { for all } p \approx 1^{+} .
$$

Therefore, (7.5) indicates a countable number of subcritical pitchfork bifurcations.

Figure 10 shows a numerical calculation for the first branch of the bifurcation diagram, where we take $l=0$ and $k=1$. Hence, in this case, the critical point is $p_{0}=4$. During each iteration of the numerical program, the calculation uses the previous results to calculate the next step, thus improving the accuracy. The step size used here is 0.001 , in the range of $p=1.7$ to $p=3.3$. Extending the range of values of $p$ closer to the critical $p_{0}=4$ proves to be difficult.

Figure 11 shows how the $p$-bifurcation diagram for the semilinear equation is expected to look like, if the branch is extended, given the numerics in Figure 10.

However the numerical analysis done in Section 6.3 has failed to provide firm evidence that Figure 11 shows how the $p$-branches behave, since we were not able to get reliable 


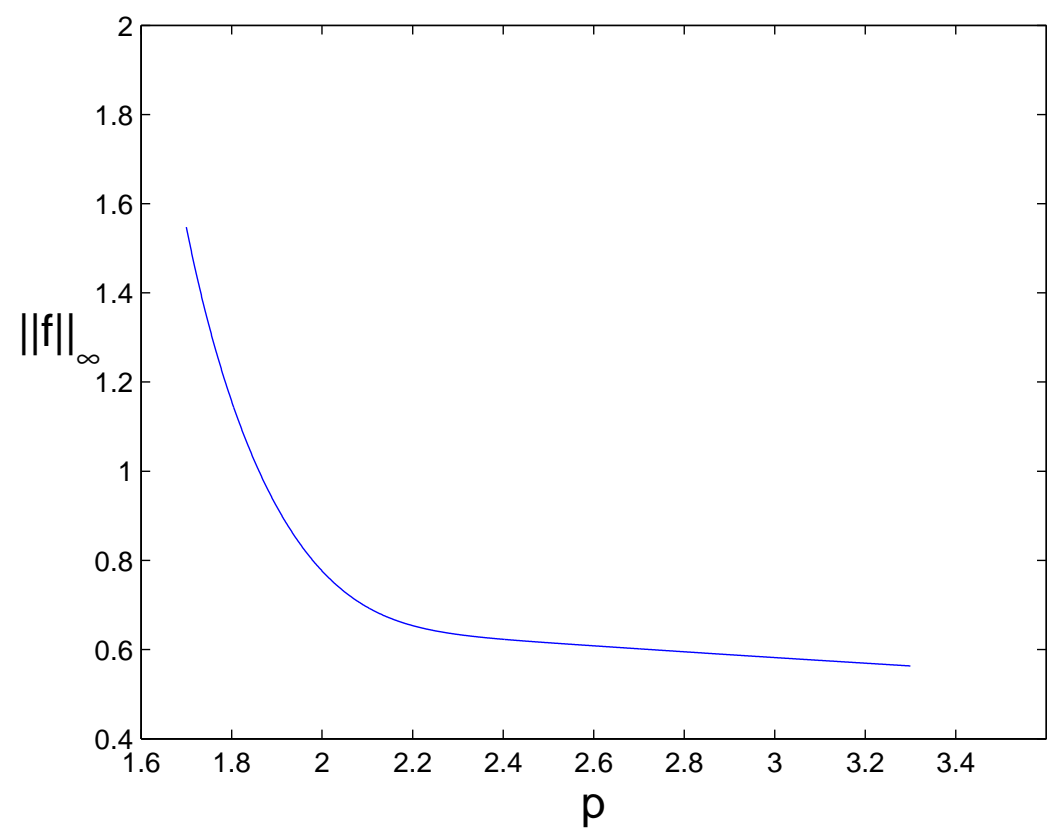

FiguRE 10. Bifurcation branch for $l=0$ and $k=1$, for the semilinear ODE (6.3).

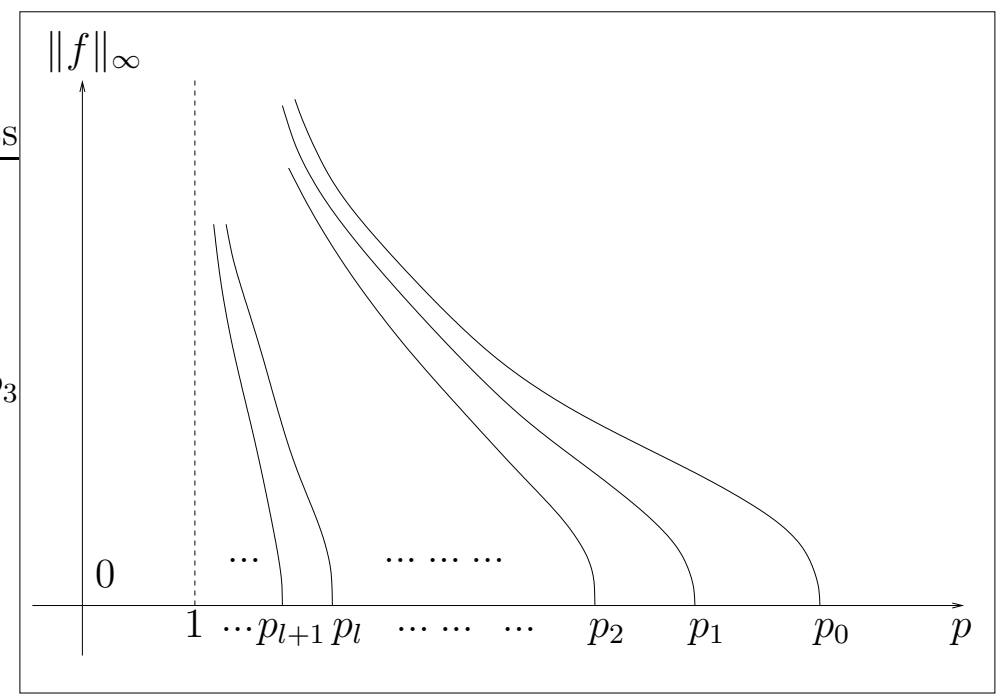

FiguRE 11. A countable family of expected p-bifurcation branches for the ODE (6.3).

numerics close to $p_{0}=4$. As before, this is related to the extremal oscillatory behaviour of similarity profiles at the right-hand side, which does not allow us using standard numerical codes of continuation with respect to the parameter $p$, i.e., numerically construct 
the so-called $p$-branches of solutions. In Figure 11, we use the analytical evidence of such bifurcations from zero at $p=p_{l}$, which also requires extra difficult mathematical justification.

\section{REFERENCES}

1. T.Ya. Azizov and I.S. Iokhvidov, Linear Operators in Spaces with Indefinite Metric, WileyInterscience, New York/Toronto, 1989.

2. C.M. Bender and S.A. Orszag, Advanced Mathematical Methods for Scientists and Engineers; Asymptotic Methods and Perturbation Theory, Springer, New York, 1999.

3. M.S. Birman and M.Z. Solomjak, Spectral Theory of Self-Adjoint Operators in Hilbert Space, D. Reidel, Dordrecht/Tokyo, 1987.

4. H. Blasius, Grenzschichten in Flüssigkeiten mit kleiner Reinbung, Z. Math. Phys. 56 (1908), 1-37.

5. J. Boussinesq, Théories des ondes et des remous qui se propagent le long d'un canal rectangulaire horizontal, en communiquant au liquide contenu dans ce canal des vitesses sensiblement pareilles de la surface au fond, J. Math. Pures Appl. 17 (1872), 55-108.

6. H. Brezis, L.A. Peletier, and D. Terman, A very singular solution of the heat equation with absorption, Arch. Rational Mech. Anal. 95 (1986), 185-209.

7. H. Cai, Dispersive smoothing effects for KdV type equations, J. Differ. Equat. 136 (1997), 191-221.

8. T. Carleman, Sur un problème d'unicité pur les systèmes d'équations aux dérivées partielles à deux variables indépendantes, Ark. Mat. Astr. Fys. 26 (1939), 1-9.

9. R. Côte, Construction of solutions to the subcritical $g K d V$ equations with a given asymptotical behaviour, J. Func. Anal. 241 (2006), 143-211.

10. W. Craig and J. Goodman, Linear dispersive equations of Airy type, J. Differ. Equat. 87 (1990), $38-61$.

11. W. Craig, T. Kappeler, and W. Strauss, Gain of regularity for equations of KdV type, Ann. Inst. H. Poincare 9 (1992), 147-186.

12. L.L. Dawson, Uniqueness properties of higher order dispersive equations, J. Differ. Equat. 236 (2007), 199-236.

13. Yu.V. Egorov, V.A. Galaktionov, V.A. Kondratiev, and S.I. Pohozaev, Global solutions of higherorder semilinear parabolic equations in the supercritical range, Adv. Differ. Equat. 9 (2004), 10091038.

14. A.V. Faminskii, On the mixed problem for quasilinear equations of the third order, J. Math. Sci. 110 (2002), 2476-2507.

15. R.S. Fernandes and V.A. Galaktionov, Eigenfunctions and very singular similarity solutions of oddorder nonlinear dispersion PDEs, submitted.

16. D. Dos Santos Ferreira, Sharp $L^{p}$ Carleman estimates and unique continuation, Duke Math. J. 129 (2005), 503-550.

17. V.A. Galaktionov, Geometric Sturmian Theory of Nonlinear Parabolic Equations and Applications, Chapman and Hall/CRC, Florida, 2004.

18. S Sturmian nodal set analysis for higher-order parabolic equations and applications, Adv. Differ. Equat. 12 (2007), 669-720.

19. _ Non-radial very singular solutions of absorption-diffusion equations with non-homogeneous potentials, Adv. Nonl. Stud. 8 (2008), 429-454. 
20. _ Nonlinear dispersion equations: smooth deformations, compactons, and extensions to higher orders, Comput. Math. Math. Phys. 48 (2008), 1823-1856 (arXiv:0902.0275).

21. V.A. Galaktionov and I.V. Kamotski, Refined scattering and Hermitiam spectral theory for linear Schrödinger eqautions with applications, in preparation.

22. V.A. Galaktionov, S.P. Kurdyumov, and A.A. Samarskiu, On asymptotic "eigenfunctions" of the Cauchy problem for a nonlinear parabolic equation, Math. USSR Sbornik 54 (1986), 421-455.

23. V.A. Galaktionov and S.I. Pohozaev, Existence and blow-up for higher-order semilinear parabolic equations: majorizing order preserving operators, Indiana Univ. Math. J. 51 (2002), 243-280.

24. _ Third-order nonlinear dispersive equations: shocks, rarefaction, and blow-up waves, Comput. Math. Math. Phys. 48 (2008), 1784-1810 (arXiv:0902.0253).

25. V.A. Galaktionov and S.R. Svirshchevskii, Exact Solutions and Invariant Subspaces of Nonlinear Partial Differential Equations in Mechanics and Physics, Chapman and Hall/CRC, Florida, 2007.

26. V.A. Galaktionov and J.L. Vázquez, A Stability Technique for Evolution Partial Differential Equations, Birkhäuser, Berlin/Boston, 2004.

27. V.A. Galaktionov and J.F. Williams, On very singular similarity solutions of a higher-order semilinear parabolic equation, Nonlinearity 17 (2004), 1075-1099.

28. T. Hoshiro, Mouree's method and smoothing properties of dispersive equations, Comm. Math. Phys. 202 (1999), 255-265.

29. I.S. Iokhvidov, Unitary operators in a space with an indefinite metric, Zap., N.I.I. Mat. i Mekh. Khar'kov Gos. Univ. Mat. Obsch. (1949), 79-86.

30. A.D. Ionescu and C.E. Kenig, $L^{p}$ Carleman inequalities and uniqueness of solutions of nonlinear Schrödinger equations, Acta. Math. 193 (2004), 193-239.

31. _ Uniqueness properties of solutions of Schrödinger equations, J. Funct. Anal. 232 (2006), 90-236.

32. S. Kamin and L.A. Peletier, Singular solutions of the heat equation with absorption, Proc. Math. Soc. 95 (1985), 205-210.

33. S. Kamin and L. Veron, Existence and uniqueness of the very singular solution of the porous media equation with absorption, J. Anal. Math. 51 (1988), 245-258.

34. T. Kato, Quasi-linear equations of evolution, with applications to partial differential equations, Spectral Theory and Differential Equations (Proc. Sympos., Dundee, 1974), pp. 27-50. Lecture Notes in Math., Vol. 448, Springer, Berlin, 1975.

35. _ On the Kortweg-de Vries equation, Manuscripta Math. 28 (1979), 89-99.

36. C.E. Kenig, G. Ponce, and L. Vega, A bilinear estimate with applications to the KdV equation, Proc. Amer. Math. Soc. 9 (1996), 573-603.

37. A.N. Kolmogorov and S.V. Fomin, Functional Analysis: Volume 1, 4th ed., Graylock, Rochester, 1957.

38. _ Elements of Theory of Functions and Functional Analysis, 4th ed., Nauka, Glav. Red. FizMat. Lit., Moscow, 1976.

39. M.G. Krein, Helical curves in an infinite-dimensional Lobachevskiy space and Lorents transformation, Izvestiya Akad. Nauk USSR, Ser. Matem. 3 (1948), 3.

40. N.A. Larkin, Modified KdV equation with a source term in a bounded domain, Math. Meth. Appl. Sci. 29 (2006), 751-765.

41. Yu.S. Ledyaev, Private communication, 2008.

42. J.L. Levandosky, Smoothing properties of nonlinear dispersive equations in two spatial dimensions, J. Differ. Equat. 175 (2001), 275-372. 
43. L. Ljusternik and V. Sobolev, Elements of Functional Analysis, Ungar Publ. Comp., New York, 1961. 44. V. Maz'ya, Sobolev Spaces, Springer-Verlag, Berlin, 1985.

45. R. Mizuhara, The initial value problem for third and fourth order dispersive equations in one space dimension, Funk. Ekvacioj 49 (2006), 1-38.

46. M.A. Naimark, Linear Differential Operators, Part I, Ungar Publ. Comp., New York, 1967.

47. L.S. Pontryagin, Hermitian operator in spaces with indefinite metric, Izvestiya Akad. Nauk USSR, Ser. Matem. 8 (1944), 243-80.

48. L. Prandtl, Über Flüssigkeitsbewegung bei sehr kleiner Reibung, In: Proc. 3rd Int. Congr., Heidelberg, 1904, Teubner, Leipzig, 1905.

49. A.A. Samarskii, V.A. Galaktionov, S.P. Kurdyumov, and A.P. Mikhailov, Blow-up in Quasilinear Parabolic Equations, Walter de Gruyter, Berlin, 1995.

50. A. Sommerfeld, Partial Differential Equations in Physics, Academic, New York, 1949.

51. W.A. Strauss, Dispersion of low-energy waves for two conservative equations, Arch. Ration. Mech. Anal. 55 (1974), 86-92.

52. C. Sturm, Mémoire sur une classe d'équations à différences partielles, J. Math. Pures Appl. 1 (1836), $373-444$.

53. H. Takuwa, Microlocal analytic smoothing effects for operators of real principal type, Osaka J. Math. 43 (2006), 13-62.

54. T. Tao, Multilinear weighted convolution of $l^{2}$ functions, and applications to nonlinear dispersive equations, Amer. J. Math. 6 (2000), 839-908.

55. X. Tao and S. Zhang, Weighted doubling properties and unique continuation theorems for the degenerate Schrödinger equations with singular potentials, J. Math. Anal. Appl. 339 (2008), 70-84.

56. M.A. Vainberg and V.A. Trenogin, Theory of Branching of Solutions of Non-Linear Equations, Noordhoff Int. Publ., Leiden, 1974.

57. J.T. Xing, An investigation into natural vibrations of fluid-structure interaction systems subject to Sommerfeld radiation condition, Acta Mech. Sin. 24 (2008), 69-82.

\section{Appendix A. "Radiation COnditions" in spectral $\left\{\mathbf{B}, \mathbf{B}^{*}\right\}$-Theory}

Here, we clarify the "radiation-type conditions" posed at infinity in the domains of operators $\mathbf{B}$ and $\mathbf{B}^{*}$, which allow the operator pair $\left\{\mathbf{B}, \mathbf{B}^{*}\right\}$ to have purely discrete spectra $\left\{-\frac{l}{2 k+1}, l \geq 0\right\}$ already detected by eigenfunction expansion of the corresponding semigroups. This serves as a description of new properties of the spaces of closure $\tilde{L}_{\rho}^{2}(\mathbb{R})$ and $\tilde{L}_{\rho^{*}}^{2}(\mathbb{R})$.

A.1. Domain $\tilde{\mathcal{D}}(\mathbf{B})$ of the linear operator B. To this end, in order for our linear ODE to be "well-posed" (i.e., with a proper number of boundary conditions at infinity), both in a mathematical sense, as well as a physical sense, we look for conditions, which must be satisfied for the eigenvalue equation:

$$
\mathbf{B} \psi_{l}(y)=\lambda_{l} \psi_{l}(y) \quad \text { in } \quad \mathbb{R} .
$$

This can be rewritten as

$$
(-1)^{k+1} \psi_{l}^{(2 k+1)}(y)+\frac{1}{2 k+1} \psi_{l}(y)+\frac{1}{2 k+1} y \psi_{l}^{\prime}(y)=\lambda_{l} \psi_{l}(y)
$$

Since the order of this ODE is $2 k+1$, it is natural that there must also be $2 k+1$ boundary conditions placed, as classic theory of ordinary differential operators suggests; see Naimark's monograph [46]. 
ELIMINATING EXPONENTIALLY GROWING BUNDLES. First consider the problem, as $y \rightarrow+\infty$. Attempting to balance leading order terms in (A.1), leads to

$$
(-1)^{k+1} \psi_{l}^{(2 k+1)}(y)+\frac{1}{2 k+1} y \psi_{l}^{\prime}(y) \sim 0
$$

As $y \rightarrow+\infty$, we have that

$$
\psi_{l}(y) \sim \mathrm{e}^{b y^{\frac{2 k+1}{2 k}}}, \quad b \in \mathbb{C}, \quad b \neq 0,
$$

hence substituting this into the above equation, yields

$$
(-1)^{k+1}\left(\frac{2 k+1}{2 k} b\right)^{2 k+1}+\frac{1}{2 k+1}\left(\frac{2 k+1}{2 k} b\right) \sim 0 \quad \Longrightarrow \quad b^{2 k} \sim(-1)^{k}\left(\frac{2 k}{2 k+1}\right)^{2 k} \frac{1}{2 k+1} .
$$

It can be seen that we end up with two cases, dependent on the value of the parameter $k$, which will determine the sign of $b^{2 k}$ and therefore its roots. Hence, for now, we ignore the term $\left(\frac{2 k}{2 k+1}\right) \frac{1}{2 k+1}$ and just look at the value of $(-1)^{k}$, assuming that $b=\left(\frac{2 k}{2 k+1}\right) \frac{1}{2 k+1} \hat{b}$.

When $k$ is even: for even values of $k$, it is noted that $\hat{b}^{2 k}=1$. Hence there must $2 k$ roots for $\hat{b}$, which are given by $\hat{b}_{m}=\mathrm{e}^{\frac{2 \pi m \mathrm{i}}{2 k}}$ for $m=0,1, \ldots, 2 k-1$.

When $k$ is odd: for odd values of $k$, there is now a negative sign, such that $\hat{b}^{2 k}=-1$. Similarly, as before, we derive $2 k$ roots, where now $\hat{b}_{m}=\mathrm{e}^{\frac{(\pi+2 \pi m) \mathrm{i}}{2 k}}$ for $m=0,1, \ldots, 2 k-1$.

For the problem to be well posed on the space $L_{\rho}^{2}$, it is important to look for roots such that there is exponential decay, rather than growth. In other words, it must satisfy the condition $\rho=\mathrm{e}^{-a y^{\alpha}}$, as $y \rightarrow+\infty$. Hence we need to eliminate any roots such that $\operatorname{Re} \hat{b}_{m}>0$. It is first noted that equality, Re $\hat{b}_{m}=0$, occurs when

$$
\frac{\pi}{2}=\left\{\begin{array}{l}
\frac{\pi m}{k} \text { for even } k \\
\frac{(\pi+2 \pi m)}{2 k} \text { for odd } k
\end{array}\right.
$$

with the same applying for $\frac{3 \pi}{2}$. For Re $\hat{b}_{m}=0$, we must have that

$$
m=\left\{\begin{array}{l}
\frac{k}{2} \quad \text { for even } k, \\
\frac{k-1}{2} \quad \text { for odd } k
\end{array} \quad \text { and } \quad m=\left\{\begin{array}{l}
\frac{3 k}{2} \text { for even } k, \\
\frac{3 k-1}{2} \text { for odd } k .
\end{array}\right.\right.
$$

Hence, in order to eliminate roots which give rise to exponential growth, the conditions placed must be such that we do not include roots such that

$$
\left\{\begin{array}{l}
m<\frac{k}{2} \quad \text { and } \quad m>\frac{3 k}{2} \quad \text { for even } k, \\
m<\frac{k-1}{2} \quad \text { and } \quad m>\frac{3 k-1}{2} \quad \text { for odd } k .
\end{array}\right.
$$

Therefore, by taking the weight

$$
\rho(y)=\mathrm{e}^{-a y^{\frac{2 k+1}{2 k}}}, \quad \text { with any sufficiently small } a>0
$$

we eliminate all exponentially growing oscillatory bundles. A sharp bound on admissible $a>0$ will be derived. Thus, we have $k-1$ conditions placed as $y \rightarrow+\infty$.

Similarly, we can do the same analysis for $y \rightarrow-\infty$. As $y \rightarrow-\infty$ in (A.2), we have that

$$
\psi(y) \sim \mathrm{e}^{b(-y)^{\frac{2 k+1}{2 k}}} \Longrightarrow b_{41}^{2 k} \sim(-1)^{k+1}\left(\frac{2 k}{2 k+1}\right) \frac{1}{2 k+1} .
$$


As expected, this only differs from the $y \rightarrow+\infty$ case by the opposite sign. This leads to

$$
\hat{b}_{m}=\left\{\begin{array}{l}
\mathrm{e}^{\frac{(\pi+2 \pi m) \mathrm{i}}{2 k}}, \quad \text { for even } k, \\
\mathrm{e}^{\frac{\pi m \mathrm{i}}{k}}, \quad \text { for odd } k, \text { for } m=0,1, \ldots, 2 k-1 .
\end{array}\right.
$$

As before, we do not want roots such that $\operatorname{Re} b_{m}>0$, where in this case

$$
\left\{\begin{array}{l}
m<\frac{k-1}{2} \text { and } m>\frac{3 k-1}{2} \text { for even } k, \\
m<\frac{k}{2} \text { and } m>\frac{3 k}{2} \quad \text { for odd } k .
\end{array}\right.
$$

Hence, for the weight (3.17), we eliminate these conditions. This leads to a further $k$ conditions, which are placed at $y \rightarrow-\infty$. All these correspond to eliminating exponentially growing asymptotic bundles that is not enough as we explain below.

Domain $\tilde{\mathcal{D}}(\mathbf{B})$ : RAdiation CONDition. As stated before, we look for $2 k+1$ conditions to be posed onto the problem. Hence there are two more conditions needed. These conditions at $y \rightarrow \pm \infty$ are known as radiation conditions. In classic problems of quantum mechanics, acoustics, and physics, the general idea behind radiation conditions is that energy sources must exactly be that and not sinks of energy. Hence all energy must be radiated from a point and scatter to infinity. We refer to the book by Sommerfeld [50, who (in 1912) first proposed radiation conditions for the Helmholtz equation, and to [57] for recent applications and references.

In our problem, the radiation conditions are rather tricky and have almost nothing to do with the classic ones. We recall that we identify those just for convenience (to verify the domain of $\mathbf{B}$ and $\mathbf{B}^{*}$ ), since the eigenfunction expansions of the semigroups, as the main tool of our asymptotic analysis, automatically include the necessary two conditions at infinity.

Remark. As is shown in [21], for the linear rescaled Schrödinger operator in $L_{\rho}^{2}\left(\mathbb{R}^{N}\right), \rho=$ $\mathrm{e}^{-a|y|^{\alpha}}, \alpha=\frac{2 m}{2 m-1}$ (a standard setting),

$$
\mathbf{B}=-\mathrm{i}(-\Delta)^{m}+\frac{1}{2 m} y \cdot \nabla+\frac{N}{2 m} I, \quad \mathbf{B}^{*}=-\mathrm{i}(-\Delta)^{m}-\frac{1}{2 m} y \cdot \nabla,
$$

the absence of a "radiation condition", accepting "oscillatory functions" at infinity only, will consequence in the fact that, in addition to the standard required spectrum $\sigma(\mathbf{B})=\left\{-\frac{|\beta|}{2 m},|\beta| \geq 0\right\}$, there appears another symmetric real one $\left\{+\frac{|\beta|}{2 m},|\beta| \geq 0\right\}$ with generalized Hermite polynomial eigenfunctions constructed as in (5.5). Fortunately, this is not the case for our operators (2.2), since the polynomials $\notin L_{\rho}^{2}$ for the weight in (3.17).

The origin of the radiation condition for $\mathbf{B}$ is as follows: Let us now balance all lower-order terms in the eigenvalue problem (A.1), so that

$$
\frac{1}{2 k+1} \psi_{l}(y)+\frac{1}{2 k+1} y \psi_{l}^{\prime}(y) \sim \lambda_{l} \psi_{l}(y) .
$$

By integration we can easily see that

$$
\psi_{l}(y) \sim A y^{(2 k+1) \lambda_{l}-1},
$$

for some constant $A$. Of course, this corresponds to the obvious root $b=0$ in the exponential expansions (A.3) and (A.5). 
We note that (A.6), is a "rational" function, unlike the exponentially oscillatory bundles in (A.3) and (A.5). For $y \rightarrow-\infty$, we know that, for any $\lambda \in \mathbb{C}$, rational solutions such as (A.6) do not belong to the space $L_{\rho}^{2}$ with the exponentially growing weight (3.17).

Thus, overall, we conclude as follows:

$$
\text { at } y=-\infty \text {, the proper weight (3.17) generates } k+1 \text { conditions. }
$$

So, this is a usual and a standard situation, so that the singular point $y=-\infty$ does not require any radiation-type condition. This is not the case for the "oscillatory" end-point $y=+\infty$.

For $y \rightarrow+\infty$, consider all complex "eigenvalues", $\lambda_{l} \in \mathbb{C}$, such that $\lambda_{l}=P+\mathrm{i} Q$, for some $P, Q \in \mathbb{R}$. From (A.6), $\psi_{l}(y)$ may now be given by

$$
\psi_{l}(y) \sim y^{\hat{P}+\mathrm{i} \hat{Q}},
$$

for $\hat{P}, \hat{Q} \in \mathbb{R}$. Hence we see that

$$
\psi_{l}(y) \sim y^{\hat{a}} \mathrm{e}^{\mathrm{i} \hat{b} \ln y} \sim y^{\hat{a}}(\cos (\hat{b} \ln y)+\mathrm{i} \sin (\hat{b} \ln y)),
$$

as $y \rightarrow+\infty$. However, we know from the asymptotic analysis that the behaviour of proper eigenfunctions is different and given by a distinct type of higher oscillatory functions:

$$
\psi_{l}(y) \sim y^{-\frac{2 k-1}{4 k}} \cos \left(y^{\frac{2 k+1}{2 k}}\right),
$$

which obviously gives a stronger oscillatory behaviour than a pure $\cos (\hat{b} \ln y)$ in (A.9). However, (A.9) admits weaker oscillatory behaviour and so we must place a condition to eliminate this behaviour. We recall the all the proper eigenfunctions being given by the generating formula $\psi_{\beta}(y)=\frac{1}{\sqrt{\beta !}} D^{\beta} F(y)$, do not contain the bundle (A.6), since the fundamental rescaled kernel $F(y)$ does not by the known divergence of the operator $\mathbf{B}$ (the equation for $F$ has been integrated once with the zero constant of integration that eliminated any trace of (A.6)); see below.

Thus, the generalized radiation condition, that is necessary for the proper discreteness of the spectrum of $\mathbf{B}$ in $L_{\rho}^{2}$, can formally be formulated as follows:

$$
\text { for the eigenvalue equation (A.1), the bundle (A.8) at } y=+\infty \text { is absent. }
$$

Then, as usual, the domain By the construction of the space of closure $\tilde{L}_{\rho}^{2}$ in Section 4.3, the condition (A.10) is assumed to be included, though we do not prove this here; cf [21] for linear rescaled Schrödinger operators.

Actually, it is easy to see that all our eigenfunctions $\left\{\psi_{l}(y)\right\}$ satisfy the above radiation condition. Indeed, $F(y) \equiv \psi_{0}(y)$ does satisfy this, by integrating once, where we have that

$$
(-1)^{k+1} F^{(2 k+1)}+\frac{1}{2 k+1}(F y)^{\prime}=0 \quad \Longrightarrow \quad(-1)^{k+1} F^{(2 k)}+\frac{1}{2 k+1} F y=C,
$$

for some constant of integration $C$. The last term precisely shows that such a rational behaviour is absent, since we have that

$$
F(y) \sim \frac{(2 k+1) C}{y}
$$

which implies that $C \equiv 0$. Then each eigenfunction $\psi_{l}(y)=\frac{1}{\sqrt{l !}} F^{(l)}(y)$ for all $l \geq 0$, also satisfies this condition. 
A.2. Radiation condition in the domain $\tilde{\mathcal{D}}\left(\mathbf{B}^{*}\right)$ of adjoint operator $\mathbf{B}^{*}$. We apply the same analysis to the adjoint operator $\mathbf{B}^{*}$, where the eigenvalue problem is now

$$
(-1)^{k+1} \psi_{l}^{*(2 k+1)}(y)-\frac{1}{2 k+1} y \psi^{* \prime}(y)=\lambda_{l} \psi^{*}(y) .
$$

Similarly, as before, we look at exponential bundles with $b \neq 0$ :

$$
\psi^{*}(y) \sim \mathrm{e}^{b y^{\frac{2 k+1}{2 k}}} \text { as } y \rightarrow+\infty, \quad \text { and } \quad \psi^{*}(y) \sim \mathrm{e}^{b|y|^{\frac{2 k+1}{2 k}}} \text { as } y \rightarrow-\infty .
$$

Balancing terms

$$
(-1)^{k+1} \psi_{l}^{*(2 k+1)}(y)=\frac{1}{2 k+1} y \psi^{* \prime}(y)+\ldots,
$$

it can easily be seen that the case for the adjoint operator $\mathbf{B}^{*}$, only differs from the linear operator $\mathbf{B}$, with respect to a change of sign. Hence it is seen that, as $y \rightarrow+\infty$ and $y \rightarrow-\infty$ respectively, for $m=0,1, \ldots, 2 k-1$,

$$
\hat{b}_{m}=\left\{\begin{array}{l}
\mathrm{e}^{\frac{(\pi+2 \pi m) \mathrm{i}}{2 k}}, \text { for even } k, \\
\mathrm{e}^{\frac{\pi m \mathrm{i}}{k}}, \text { for odd } k,
\end{array} \quad \text { and } \quad \hat{b}_{m}=\left\{\begin{array}{l}
\mathrm{e}^{\frac{\pi m \mathrm{i}}{k}}, \text { for even } k, \\
\mathrm{e}^{\frac{(\pi+2 \pi m) \mathrm{i}}{2 k}}, \text { for odd } k .
\end{array}\right.\right.
$$

It is noted that we look at the problem in the space $L_{\rho^{*}}^{2}$, with weight

$$
\rho^{*}(y)=\mathrm{e}^{-a|y|^{\frac{2 k+1}{2 k}}}, \quad a>0 \text { is small enough. }
$$

Hence, for this weight, for $y \rightarrow+\infty$, we eliminate the roots such that

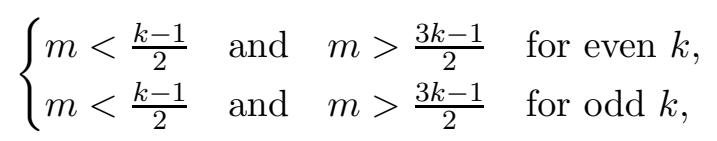

and for $y \rightarrow-\infty$

$$
\left\{\begin{array}{l}
m<\frac{k}{2} \quad \text { and } \quad m>\frac{3 k}{2} \quad \text { for even } k, \\
m<\frac{k-1}{2} \quad \text { and } \quad m>\frac{3 k-1}{2} \quad \text { for odd } k .
\end{array}\right.
$$

These give $2 k-1$ conditions in total.

Whilst before balancing the rest of the terms will lead to our radiation conditions, this is not possible to do this in similar lines (as for $\mathbf{B}$ ) in the case for $\mathbf{B}^{*}$. Note that, on integration, there holds:

$$
-\frac{1}{2 k+1} y \psi^{* \prime}(y) \sim \lambda_{l} \psi^{*}(y) \quad \Longrightarrow \quad \psi_{l}^{*}(y) \sim A_{0} y^{-(2 k+1) \lambda_{l}}
$$

for some constant $A_{0}$. However, this behaviour is perfectly acceptable in $L_{\rho^{*}}^{2}$, and it is satisfied by the polynomial adjoint eigenfunctions $\left\{\psi_{l}^{*}(y)\right\}$, which are our generalized Hermite polynomials.

Instead we look for the remaining two conditions for the problem as $y \rightarrow-\infty$. In light of the definition of $\mathbf{B}^{*}$, we assume to have another type of radiation condition, which for convenience we denote as the "Adjoint Radiation Condition", such that we

$$
\text { exclude two bundles with "maximal" oscillatory components at } y=-\infty \text {. }
$$

Again, this extra radiation condition makes the total number of conditions to be equal to $2 k+1$, which is the differential order of the operator $\mathbf{B}^{*}$, so that the eigenvalue problem becomes algebraically consistent [46]. In other words, we have an algebraic inhomogeneous system of $2 k+1$ equations with analytic coefficients with $2 k+1$ unknowns. Such systems do not have 
more than a countable set of solutions, which are eigenvalues of $\mathbf{B}^{*}$, which is defined in such a way.

Hence, we now restrict those roots such that Re $a_{m}=0$. Hence the conditions, as $y \rightarrow+\infty$ are now given by the following distribution of the acceptable coefficients $\left\{b_{m}\right\}$ :

$$
\left\{\begin{array}{l}
m \leq \frac{k}{2} \quad \text { and } \quad m \geq \frac{3 k}{2} \quad \text { for even } k, \\
m \leq \frac{k-1}{2} \quad \text { and } m \geq \frac{3 k-1}{2} \quad \text { for odd } k .
\end{array}\right.
$$

A.3. Calculations for the weights, $\rho(y)$ and $\rho^{*}(y)$. We now determine the sharp distance between the principle root $b_{m_{c}}$ such that $\operatorname{Re} b_{m_{c}}=0$ and the previous root $b_{m_{c}-1}$, where $\operatorname{Re} b_{m_{c}-1}>0$. In doing so we may find our weight $\rho(y)$ such that it cuts off all unwanted roots, for which $\operatorname{Re} b>0$.

First consider the case as $y \rightarrow+\infty$. For Re $b_{m_{c}}=0$, it is known that there is a root here and this is given by

$$
b_{m_{c}}=d_{k} \mathrm{i}, \quad \text { where } \quad d_{k}=2 k\left(\frac{1}{2 k+1}\right)^{\frac{2 k+1}{2 k}} \quad \text { and } \quad m_{c}= \begin{cases}\frac{k}{2} & \text { for even } k \\ \frac{k-1}{2} & \text { for odd } k .\end{cases}
$$

Hence, the root $m_{c}-1$ is given by

$$
m_{c}-1= \begin{cases}\frac{k-2}{2} & \text { for even } k \\ \frac{k-3}{2} & \text { for odd } k\end{cases}
$$

This yields

$$
b_{m_{c}-1}=\left(\cos \frac{(k-2) \pi}{2 k}+\mathrm{i} \sin \frac{(k-2) \pi}{2 k}\right) d_{k},
$$

for all $k$. Hence the distance between the two roots (in the real axis) is

$$
d=d_{k} \cos \frac{(k-2) \pi}{2 k}>0 .
$$

For $y \rightarrow-\infty$, we do not have any roots $\operatorname{Re} b_{m_{c}}=0$, for any $k$. However we look at the distance between the real axis and the next root such that $\operatorname{Re} b>0$.

In this case we find the distance between the real axis and roots such that

$$
m_{c}-1=\left\lfloor\frac{k-1}{2}\right\rfloor
$$

for all $k$. Hence we have that

$$
b_{m_{c}-1}=\left\{\begin{array}{l}
d_{k}\left[\cos \left(\left\lfloor\frac{k-1}{2}\right\rfloor \frac{\pi}{k}+\frac{\pi}{2 k}\right)+\mathrm{i} \sin \left(\left\lfloor\frac{k-1}{2}\right\rfloor \frac{\pi}{k}+\frac{\pi}{2 k}\right)\right] \text { for even } k, \\
d_{k}\left[\cos \left(\frac{k-1}{2} \frac{\pi}{k}\right)+\mathrm{i} \sin \left(\frac{k-1}{2} \frac{\pi}{k}\right)\right] \text { for odd } k .
\end{array}\right.
$$

This gives the distance between this root and the real axis

$$
d=\left\{\begin{array}{l}
d_{k} \cos \left(\left\lfloor\frac{k-1}{2}\right\rfloor \frac{\pi}{k}+\frac{\pi}{2 k}\right) \quad \text { for even } k \\
d_{k} \cos \left(\frac{k-1}{2} \frac{\pi}{k}\right) \text { for odd } k
\end{array}\right.
$$

This characterises our weighted space $L_{\rho}^{2}(\mathbb{R})$, with the exponential weight

$$
\rho(y)= \begin{cases}\mathrm{e}^{a|y|^{\frac{2 k+1}{2 k}}} & \text { for } y \leq-1 \\ \mathrm{e}^{-a y^{\frac{2 k+1}{2 k}}} & \text { for } y \geq 1\end{cases}
$$


where $a \in(0,2 d)$.

Similarly we can find the weight $\rho^{*}(y)$. We note that the calculations are exactly the same as in the case for $\rho(y)$, except a difference in sign when calculating the roots. This leads to

$$
d=\left\{\begin{array}{l}
d_{k} \cos \left(\left\lfloor\frac{k-1}{2}\right\rfloor \frac{\pi}{k}+\frac{\pi}{2 k}\right) \quad \text { for even } k \\
d_{k} \cos \left(\frac{k-1}{2} \frac{\pi}{k}\right) \text { for odd } k
\end{array}\right.
$$

as $y \rightarrow+\infty$ and

$$
d=\left\{\begin{array}{l}
d_{k} \cos \left(\frac{k-2}{2} \frac{\pi}{k}\right) \quad \text { for even } k, \\
d_{k} \cos \left(\left\lfloor\frac{k-2}{2}\right\rfloor \frac{\pi}{k}+\frac{\pi}{2 k}\right) \text { for odd } k,
\end{array}\right.
$$

as $y \rightarrow-\infty$. Here the weight $\rho^{*}(y)$ is defined as in (5.4).

\section{Appendix B. Estimates on the FUndamental KERNEL AND MAJORIZING OPERATOR}

Here, we develop some ideas concerning majorizing-comparison issues for the odd-order operators involved, which literally do not have any connections with the standard Maximum Principle.

B.1. Estimates of the rescaled kernel. Recalling our estimate given by (2.11), we now look to estimate our rescaled fundamental kernel $F(y)$ by

$$
|F(y)| \leq \bar{D} \bar{F}(y), \quad \text { where } \quad \bar{F}(y)>0 \quad \text { and } \quad \int \bar{F}(y)=1 .
$$

Here $\bar{D}$ is a normalisation constant, obviously satisfying $\bar{D}>1$. There exists infinitely many functions which satisfy (B.1), but since our kernel $F$ is changing sign, it is not possible to find an optimal analytic function

$$
\bar{F}_{\text {opt }}(y)=\omega_{1}|F(y)|,
$$

where $\omega_{1}>0$ is a normalisation constant, such that

$$
\int \bar{F}_{\text {opt }}=1 \quad \Longrightarrow \quad \omega_{1}=\left(\int|F|\right)^{-1}>1 \text {. }
$$

However, we can find an analytical approximation of $\bar{F}_{\text {opt }}$ such that (B.1) is satisfied, but is non-optimal. One such function is given by

$$
\bar{F}_{*}(y)=\omega_{1}\left(1+y^{2}\right)^{-\frac{(2 k-1)}{8 k}}\left(\frac{1}{1+\mathrm{e}^{-y}}+\frac{1}{1+\mathrm{e}^{y}} \mathrm{e}^{-a\left(1+y^{2}\right)^{\frac{\alpha}{2}}}\right) .
$$

A sketch of the function $\bar{F}_{*}$ is shown by Figure B.1 and comparison with the numerics in Section 2.3 shows how the function may be an upper bound for $F(y)$.

By using the asymptotic analysis set out in Section 2.2, we can also find that all higher order derivatives are estimated by

$$
\left|D^{\beta} F(y)\right| \leq\left\{\begin{array}{l}
\bar{c}^{\beta} \beta^{\frac{(\alpha-1)}{\alpha} \beta} \mathrm{e}^{-a|y|^{\alpha}} \quad \text { for } \quad y \leq-1, \\
\bar{c}^{\beta} y^{\frac{\beta}{2 k}} \text { for } y \geq 1
\end{array}\right.
$$

where $\bar{c}$ is dependent on $k$ only. 


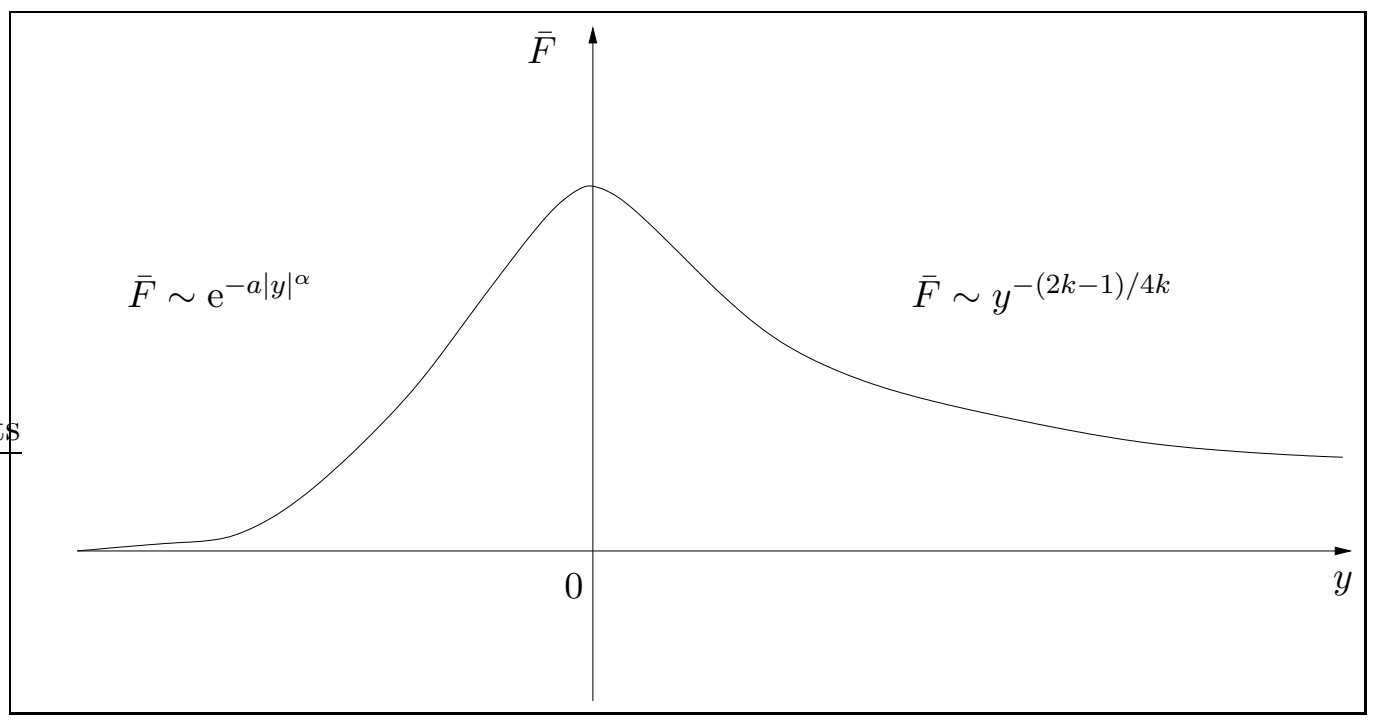

FiguRE 12. Majorizing kernel $\bar{F}(y)$.

B.2. Majorizing kernel and spectral properties. We now introduce the positive majorizing "fundamental solution"

$$
\bar{b}(x, t)=t^{-\frac{1}{2 k+1}} \bar{F}(y), \quad y=x / t^{\frac{1}{2 k+1}},
$$

with the majorizing $\bar{F}$ relative to the kernel $F$ of $b(x, t)$ in (2.1). We then end up with a formal integral evolution equation written in the standard form

$$
\bar{u}_{t}=\overline{\mathbf{A}}(t) \bar{u},
$$

for some linear operator $\overline{\mathbf{A}}(t)$, with the "fundamental solution" $\bar{b}(x, t)$. This formal nonautonomous (in time $t$ ) evolution equation is understood in the sense that the Cauchy problem for (B.3) with initial data

$$
\bar{u}(x, 0)=\bar{u}_{0}(x) \geq 0 \quad \text { in } \quad \mathbb{R}
$$

is given by the convolution

$$
\bar{u}(x, t)=\overline{\mathbf{M}}(t) \bar{u}_{0}(x) \equiv \bar{b}(t) * \bar{u}_{0}=t^{-\frac{1}{2 k+1}} \int \bar{F}\left((x-z) t^{-\frac{1}{2 k+1}}\right) \bar{u}_{0}(z) \mathrm{d} z .
$$

It can be seen from this, that for general kernels $\bar{F}$, majorizing semigroups do not exist, so equation (B.3) does not admit translation in time. This defines the corresponding majorizing integral equation. For higher-order parabolic (poly-harmonic) equation, the idea of majorizing integral operators was introduced and applied in blow-up studied in [23].

As before, let us now introduce rescaled variables

$$
\bar{u}(x, t)=t^{-\frac{1}{2 k+1}} \bar{w}(y, \tau), \quad y=x t^{-\frac{1}{2 k+1}}, \quad \tau=\ln t .
$$

Hence from convolution in (B.5)

$$
\bar{w}(y, \tau) \equiv \int \bar{F}\left(y-z \mathrm{e}^{-\frac{\tau}{2 k+1}}\right) \bar{u}_{0}(z) \mathrm{d} z .
$$


Using Taylor's power series we formally obtain

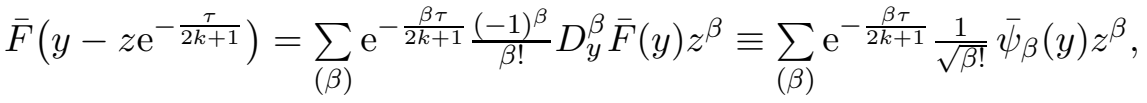

$$
\begin{aligned}
& \text { where } \quad \bar{\psi}_{\beta}(y)=\frac{(-1)}{\sqrt{\beta !}}^{\beta} D_{y}^{\beta} \bar{F}(y) .
\end{aligned}
$$

The convergence of (B.6) on bounded intervals is guaranteed by the estimates of $D_{y}^{\beta} \bar{F}(y)$ given in (B.2). A proper convergence in $L_{\rho}^{2}$ can be also included as above.

The solution can then be represented by

$$
\bar{w}(y, \tau)=\sum_{(\beta)} \mathrm{e}^{-\frac{\beta \tau}{2 k+1}} \bar{M}_{\beta}\left(u_{0}\right) \bar{\psi}_{\beta}(y), \bar{\lambda}_{\beta}=-\frac{\beta}{2 k+1}, \text { where } \bar{M}_{\beta}\left(\bar{u}_{0}\right)=\frac{1}{\sqrt{\beta !}} \int_{\mathbb{R}} z^{\beta} \bar{u}_{0}(z) \mathrm{d} z
$$

are the corresponding moments of the initial data.

Proposition B.1. There exists some formal operator $\overline{\mathbf{B}}$, such that

$$
\bar{w}_{\tau}=\overline{\mathbf{B}} \bar{w}
$$

and this induces the majorizing semigroup $\left\{\mathrm{e}^{\overline{\mathbf{B}} \tau}\right\}$, formulated by the rescaled variables.

It follows from (3.10) that $\overline{\mathbf{B}}$ has point spectrum given by $\sigma_{p}(\overline{\mathbf{B}})=\left\{\bar{\lambda}_{\beta}\right\}$ and corresponding eigenfunctions are thus given by $\bar{\Phi}=\left\{\bar{\psi}_{\beta}\right\}$. With a standard definition of the space of $\bar{\Phi}$-closure $\tilde{L}_{\rho}^{2}$ (see Section 4.3), this becomes the only spectrum of $\overline{\mathbf{B}}$.

In order to find a full expansion form of the majorizing semigroup, we once again perform another rescaling given by

$$
\bar{u}=(1+t)^{-\frac{1}{2 k+1}} \bar{w}, \quad y=x(1+t)^{-\frac{1}{2 k+1}}, \quad \tau=\ln (1+t): \mathbb{R}_{+} \rightarrow \mathbb{R}_{+} .
$$

Then rescaling the convolution we obtain

$$
\bar{w}(y, \tau)=\mathrm{e}^{\overline{\mathbf{B}} \tau} \bar{u}_{0} \equiv\left(1-\mathrm{e}^{-\tau}\right)^{-\frac{1}{2 k+1}} \int_{\mathbb{R}} \bar{F}\left(\left(y-z \mathrm{e}^{-\frac{\tau}{2 k+1}}\right)\left(1-\mathrm{e}^{-\tau}\right)^{-\frac{1}{2 k+1}}\right) \bar{w}_{0}(z) \mathrm{d} z .
$$

Using Taylor expansions, we find that the solution is given by

$$
\begin{gathered}
\bar{w}(y, \tau)=\left(1-\mathrm{e}^{-\tau}\right)^{-\frac{1}{2 k+1}} \sum_{(\mu, \nu)} \frac{(-1)}{\mu ! \nu !}{ }^{\mu} D^{\nu} \bar{F}(0) \frac{1}{(\nu-\mu) !} y^{\nu-\mu}\left(\mathrm{e}^{\tau}-1\right)^{-\frac{\mu}{2 k+1}} \\
\times\left(1-\mathrm{e}^{-\tau}\right)^{-\frac{\nu}{2 k+1}} \int_{\mathbb{R}} z^{\mu} \bar{w}_{0}(z) \mathrm{d} z .
\end{gathered}
$$

This again shows the discrete spectrum, as well as some traces of generalized Hermite polynomials $\left\{\bar{\psi}_{\beta}^{*}\right\}$.

The adjoint operator $\overline{\mathbf{B}}^{*}$ with such polynomial eigenfunctions $\left\{\bar{\psi}_{\beta}^{*}\right\}$ occurs if we use the blowup scaling

$$
\bar{u}(x, t)=\bar{w}(y, \tau), \quad y=x(1-t)^{-\frac{1}{2 k+1}}, \quad \tau=-\ln (1-t) .
$$

We thus obtain

$$
\bar{w}(y, \tau)=\left(1-\mathrm{e}^{-\tau}\right) \int_{\mathbb{R}} \bar{F}\left(\left(y \mathrm{e}^{-\frac{\tau}{2 k+1}}-z\right)\left(1-\mathrm{e}^{-\tau}\right)^{-\frac{1}{2 k+1}}\right) \bar{w}_{0}(z) \mathrm{d} z .
$$


Using Taylor expansion yields

$$
\begin{aligned}
\bar{w}(y, \tau)=\left(1-\mathrm{e}^{-\tau}\right)^{-\frac{1}{2 k+1}} \sum_{(\beta, \nu)} & \frac{(-1)^{\beta}}{\beta ! \nu !}\left(1-\mathrm{e}^{-\tau}\right)^{-\frac{\beta}{2 k+1}}\left(\mathrm{e}^{\tau}-1\right)^{-\frac{\nu}{2 k+1}} \\
& \times D^{\nu} F(0) \frac{1}{(\nu-\beta) !} y^{\nu-\mu} \int_{\mathbb{R}} z^{\beta} \bar{w}_{0}(z) \mathrm{d} z .
\end{aligned}
$$

As above, further expanding of exponential terms here leads to the eigenfunction expansion, which determines finite generalized Hermite polynomials. This representation is rather technical and we do not present and analyze it since do not aim using those polynomials in what follows. Our main application of the majorizing operators is as follows:

B.3. Comparison with majorizing problem. We look to see how the majorizing kernel relates to our real solution $u(x, t)$. By looking at the convolution (3.1) for the linear PDE, we can easily see that

$$
|u(x, t)| \leq|b(t)| *\left|u_{0}(x)\right| .
$$

Now looking at our estimate of the majorizing kernel, $\bar{b}(x, t)$, we have that

$$
|u(x, t)| \leq \bar{D} \bar{b}(x, t) *\left|u_{0}(x)\right| \leq \bar{b}(t) * \bar{u}_{0}(x),
$$

where we have to assume the following inequality for initial data:

$$
\bar{D}\left|u_{0}(x)\right| \leq \bar{u}_{0}(x) \quad \text { in } \quad \mathbb{R} .
$$

Proposition B.2. If (B.4) and (B.7) hold then

$$
|u(x, t)| \leq \bar{u}(x, t) \quad \text { in } \quad \mathbb{R} \times \mathbb{R}_{+} .
$$

We introduce the majorizing kernel, since the structural behaviour of self-similar solutions of the majorizing evolution equation describes essential features of the solutions to the original PDE. Since we have estimated our solution, it can be possible to find properties of the real solution, which otherwise would be more difficult to achieve.

The linear semigroup for $\mathbf{B}$ is not order-preserving, since the kernel $F$ is oscillatory. Therefore we must have $\bar{D}>1$, which gives the order deficiency of the linear operator $\mathbf{B}$ and of the linear convolution operator $\bar{M}(t)$, given in (B.5). The actual defect is, in fact, characterized by $\bar{D}-1>0$. The linear semigroup for $\mathbf{B}$ would only be order-preserving if $F$ did not change sign, since then we would have that $F=\bar{F}$ and so we would have $\bar{D}=1$ and so the defect would be zero. As we have shown, this is not possible for any $k \geq 1$.

Department of Mathematical Sciences, University of Bath, Bath BA2 7Ay, UK

E-mail address: vag@maths.bath.ac.uk

Department of Mathematical Sciences, University of Bath, Bath BA2 7AY, UK

E-mail address: rsf21@maths.bath.ac.uk 